\title{
Temporal Characterization of Microglia-Associated Pro- and Anti-Inflammatory Genes in a Neonatal Inflammation-Sensitized Hypoxic-Ischemic Brain Injury Model
}

\author{
Maria E. Bernis, ${ }^{1,2}$ Yvonne Schleehuber, ${ }^{1,2}$ Margit Zweyer, ${ }^{1,2}$ Elke Maes, ${ }^{1,2}$ \\ Ursula Felderhoff-Müser, ${ }^{3}$ Daniel Picard, ${ }^{4,5}$ and Hemmen Sabir ${ }_{(D)}^{1,2,3}$ \\ ${ }^{1}$ Department of Neonatology and Pediatric Intensive Care, Children's Hospital University of Bonn, Bonn, Germany \\ ${ }^{2}$ Deutsche Zentrum für Neurodegenerative Erkrankungen (DZNE), Bonn, Germany \\ ${ }^{3}$ Department of Pediatrics I, Neonatology and Experimental Perinatal Neurosciences, University Hospital Essen, \\ University Duisburg-Essen, Essen, Germany \\ ${ }^{4}$ Division of Pediatric Neuro-Oncogenomics, German Consortium for Translational Cancer Research (DKTK), Partner site Essen/ \\ Düsseldorf, Düsseldorf, Germany \\ ${ }^{5}$ Department of Pediatric Oncology, Hematology and Clinical Immunology, Medical Faculty, Heinrich Heine University, \\ Düsseldorf, Germany
}

Correspondence should be addressed to Hemmen Sabir; hemmen.sabir@dzne.de

Received 22 November 2021; Revised 22 December 2021; Accepted 27 January 2022; Published 2 March 2022

Academic Editor: Stefanie Endesfelder

Copyright (c) 2022 Maria E. Bernis et al. This is an open access article distributed under the Creative Commons Attribution License, which permits unrestricted use, distribution, and reproduction in any medium, provided the original work is properly cited.

\begin{abstract}
Hypoxic-ischemic encephalopathy (HIE) mainly affects preterm and term newborns, leading to a high risk of brain damage. Coexisting infection/inflammation and birth asphyxia are key factors associated with intracerebral increase of proinflammatory cytokines linked to HIE. Microglia are key mediators of inflammation during perinatal brain injury, characterized by their phenotypic plasticity, which may facilitate their participation in both the progression and resolution of injury-induced inflammation. The purpose of this study was to investigate the temporal expression of genes associated with pro- and antiinflammatory cytokines as well as the nucleotide-binding domain, leucine-rich repeat protein (NLRP-3) inflammasome from microglia cells. For this purpose, we used our established neonatal rat model of inflammation-sensitized hypoxic-ischemic (HI) brain injury in seven-day-old rats. We assessed gene expression profiles of 11 cytokines and for NLRP-3 using real-time PCR from sorted CD11b/c microglia of brain samples at different time points ( $3.5 \mathrm{~h}$ after LPS injection and $0,5,24,48$, and 72 hours post HI) following different treatments: vehicle, E. coli lipopolysaccharide (LPS), vehicle/HI, and LPS/HI. Our results showed that microglia are early key mediators of the inflammatory response and exacerbate the inflammatory response following HI, polarizing into a predominant proinflammatory M1 phenotype in the early hours post HI. The brains only exposed to HI showed a delay in the expression of proinflammatory cytokines. We also demonstrated that NLRP-3 plays a role in the inflammatory resolution with a high expression after HI insult. The combination of both, a preinfection/inflammation condition and hypoxia-ischemia, resulted in a higher proinflammatory cytokine storm, highlighting the significant contribution of acute inflammation sensitizing prior to a hypoxic insult on the severity of perinatal brain damage.
\end{abstract}

\section{Introduction}

Neonatal hypoxic-ischemic encephalopathy (HIE) is a type of brain injury due to a lack of oxygen and blood flow to the brain during the neonatal period [1]. HIE is a leading cause of neonatal mortality, and it is associated with a variety of life-long morbidities [2,3]. HIE has an incidence of approximately 1.5 cases per 1000 live births in developed countries and 10-20 per 1000 live births in low- and middle-income countries [4-6]. The etiology of HIE is multifactorial; 
antenatal infection/inflammation (i.e., chorioamnionitis), hypoxia-ischemia, and various postnatal injurious triggers contribute to the severity of the brain injury and adverse outcome $[2,3,7-10]$. Therapeutic hypothermia (TH) is the current standard treatment for newborns with $\mathrm{HIE}[4,11]$. TH reduces the risk of death or adverse long-term neurodevelopmental outcome by $15 \%$, leading to improved outcomes following moderate HIE. However, as shown in randomized controlled trials, only $50 \%$ of cooled asphyxiated newborns benefit from cooling treatment [4]; and $\mathrm{TH}$ has not demonstrated improvement in outcomes related to severe HIE and neonatal encephalopathy in the context of perinatal infection [12-16].

Several studies in animal models, where lipopolysaccharide (LPS) was used as infection simulants, showed that acute infections following inflammation before a second insult such as hypoxia-ischemia (HI) (termed "sensitization") exacerbate brain injury [15, 17-26]. Previous studies from our laboratory demonstrated that $\mathrm{TH}$, showing significant neuroprotection after HI brain injury in our animal model, is not neuroprotective in our neonatal animal model of inflammation-sensitized HI brain injury [15, 17].

Exposure to multiple inflammatory perinatal triggers contributes to the development of a self-perpetuating cascade of peripheral and cerebral immune-inflammation responses that play a critical role in HI brain injury [27]. Microglia are specialized immune cells of the central nervous system (CNS) [28-30]. During an insult, such as LPSassociated inflammation or HI [31], microglia are rapidly activated, changing their morphology into a motile "amoeboid" state, proliferate, and migrate to the damage regions, where they release a variety of cytokines, chemokines, reactive oxygen species, and excitotoxicity molecules [32, 33]. Studies show that depletion of microglia in models of HIE increases brain injury, indicating the additional essential role of microglia for tissue repair $[34,35]$. Two distinct polarization states of activated microglial cells have been discovered. Depending on microenvironmental cues, microglia can rapidly change their phenotype to proinflammatory (type M1) cells or anti-inflammatory (type M2) cells [36, 37]. The polarization of M1/M2 microglia does not seem to follow strict differentiation and can differ depending on the different levels of brain maturity and vulnerability to aggression due to regional and age-specific metabolic needs [38, 39].

The nucleotide-binding domain, leucine-rich repeat protein (NLRP-3) inflammasome, is highly involved in neonatal brain injury either due to LPS or HI [40]. NLRP-3 is responsible for the cleavage of interleukin IL-18 and IL-1beta from its preforms. As shown, the vulnerability of the neonatal brain to LPS or HI is IL-18 and IL-1beta dependent, predominantly leading to microglia activation [41].

We have previously shown in our inflammationsensitized model of HI brain injury a significant increase in brain area loss and neuronal injury $24 \mathrm{~h}$ post HI [42]. Additionally, we have shown that microglia polarize into a predominantly proinflammatory phenotype $24 \mathrm{~h}$ post $\mathrm{HI}$, and we showed an increase in the gene expression of NLRP-3 $[42,43]$. However, we have only analyzed one time point so far- $24 \mathrm{~h}$ post HI. The time-dependent expression of pro- and anti-inflammatory cytokines regulated by microglia has yet not been investigated.

Therefore, we used LPS, a component of the cell walls of gram-negative bacteria, and a potent endotoxin, to presensitize the brain and to simulate perinatal infection and inflammation. We hypothesized that the combination of LPS and $\mathrm{HI}$ would exacerbate brain injury compared to $\mathrm{HI}$ alone, with an early increase in the expression pattern of proinflammatory cytokines as well as the activation of the NLRP3 inflammasome.

\section{Material and Methods}

2.1. Animals and Experimental Procedure. All animal experiments were performed in accordance with the Animal Protection Committee of the North Rhine-Westphalia State Environment Agency (LANUV), Germany, and following the ARRIVE guidelines. The study was performed using 7day old (P7) Wistar rat pups of both genders in all our experiments. All pups were kept at the central animal laboratory of the Deutsche Zentrum für Neurodegenerative Erkrankungen (DZNE) Bonn, Germany, with a 12:12 h dark/ light cycle at an environmental temperature of $21^{\circ} \mathrm{C}$ with food and water ad libitum. As previously described, all animals for each treatment were randomized across litter, sex, and weight before the experiments commenced, and all following experiments and analysis were performed by observers blinded to the different treatments [15, 17]. A total of 244 animals were used (129 females and 115 males) and randomized to different treatment groups. 160 rat pups survived our inflammation-sensitized HI insult. Mortality was highest in the LPS/HI group as it has been expected and reported $[15,17,42]$. Four groups were used and brains analyzed at different time points: vehicle ( $n=5$ per time point), LPS ( $n=5$ per time point), vehicle/HI $(n=10$ per time point), and LPS/HI ( $n=10$ per time point). Temperature was monitored in "sentinel" rat pups not allocate to the different treatment groups during experimental procedures. During all the experiment, temperature of the rat pups was controlled by the sentinel pup via a rectal probe (IT-21, Physitemp Instruments, Clifton, NJ, USA) controlling a mat (CritiCool, MTRE, Yavne, Israel). The sentinel pup kept the nesting temperature of P7 rat pups [19] or treatment temperatures during experiments (see below). All rats from the LPS and LPS/HI group received a single intraperitoneal (i.p.) injection of LPS solution (Escherichia coli O55:B5, Sigma; $0.1 \mathrm{mg} / \mathrm{kg})$. The vehicle and vehicle/HI groups received a single i.p. injection of saline $(\mathrm{NaCl} 0.9 \%)$. After a delay of $3.5 \mathrm{~h}$ post injection, while animals were kept with their dams, and the vehicle/HI and LPS/HI animals were exposed to our model of HI brain injury as previously described $[15,17]$. Briefly, under general isoflurane anesthesia, the left common carotid artery was ligated and cut. Within $3 \mathrm{~h}$, the pups were subjected to $8 \% \mathrm{O}_{2}$ for $50 \mathrm{~min}$ at a rectal temperature $\left(T_{\text {rectal }}\right)$ of $36^{\circ} \mathrm{C}$, resulting in mild $\mathrm{HI}$ brain injury $[15,17]$. Immediately after $\mathrm{HI}$, pups were kept at $T_{\text {rectal }}$ of $37.0^{\circ} \mathrm{C}$ for $5 \mathrm{~h}$, representing the normothermia treatment group in our previous experiments [15-17]. Following the treatment period, the pups were returned to their 


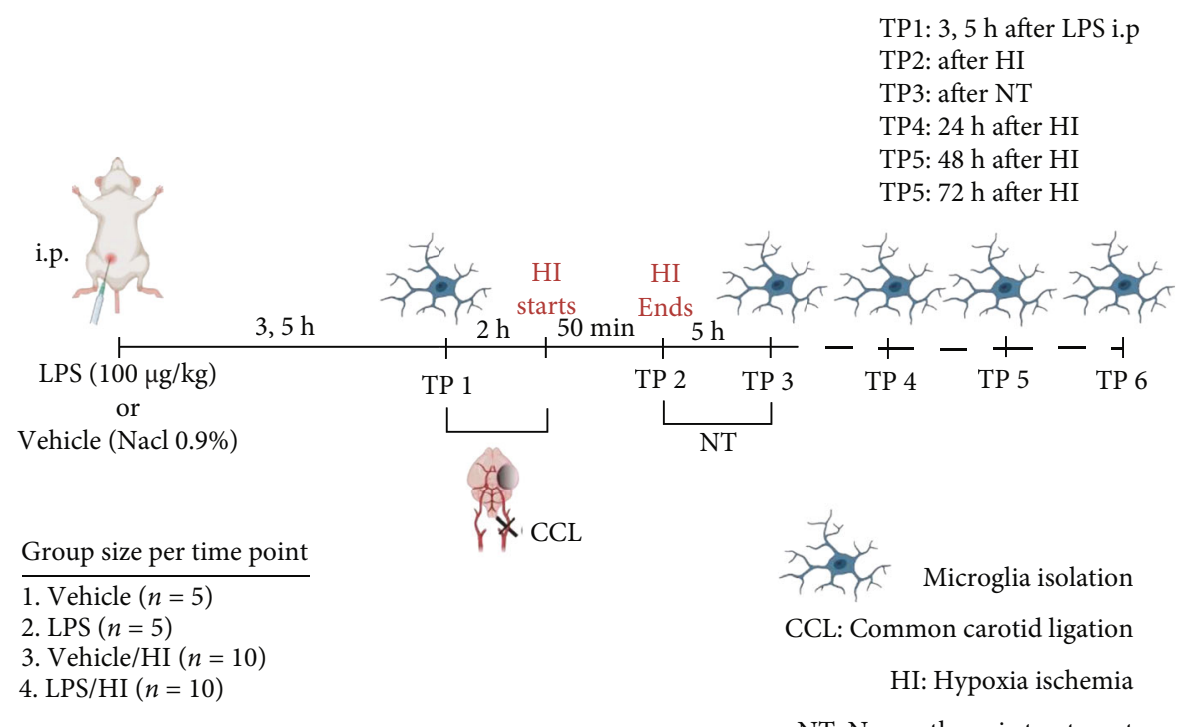

Figure 1: Experimental design: 7-day-old rats (P7) were randomized into 4 different treatment groups. Rats were either injected intraperitoneally (i.p.) with vehicle $(\mathrm{NaCl} 0.9 \%)$ or LPS $(100 \mu \mathrm{g} / \mathrm{mL}) 3.5 \mathrm{~h}$ before exposure to unilateral ligation of the left common carotid artery. Following ligation, rats were exposed to hypoxia treatment ( $\mathrm{HI})\left(8 \% \mathrm{O}_{2}-36^{\circ} \mathrm{C}\right)$ for 50 min before being treated with normothermia (NT) $\left(37^{\circ} \mathrm{C}\right)$ for $5 \mathrm{~h}$ and thereafter returned to their dam. Different time points were analyzed. TP1 is $3.5 \mathrm{~h}$ before ligation. TP2 is immediately after HI. TP3 is immediately after NT. TP4, TP5, and TP6 are 24, 48, and $72 \mathrm{~h}$ after HT, respectively. The brain was extracted and microglia isolated via magnetic cell sorting for CD11 b/c-positive cell. Figure created with http://BioRender.com.

dam. The pups were sacrificed at different time points (Figure 1). For all the pups at the first time point (TP1; see Figure 1), no ligation was performed, nor exposure to HI.

2.2. Immunohistochemistry. For histological analysis, the pups were sacrificed at TP4 (24h after HI). Following transcardial perfusion with phosphate-buffered saline (PBS), followed by $4 \%$ paraformaldehyde (Sigma-Aldrich), the brains were postfixed in $4 \%$ paraformaldehyde overnight at $4^{\circ} \mathrm{C}$ and embedded in paraffin. Immunohistochemistry was performed as previously described [44, 45]. We used TP4 for our immunohistochemistry since at TP5 and TP6 (48 and $72 \mathrm{~h}$ post $\mathrm{HI}$, respectively), the cortex and hippocampal areas in the LPS/HI were severely affected (data not shown). After deparaffinization, $10 \mu \mathrm{m}$ coronal sections $(-3.8 \pm 0.7$ $\mathrm{mm}$ from bregma) were rehydrated. Antigen retrieval was performed in preheated PBS $1 \mathrm{x}$ for $7 \mathrm{~min}$ following permeabilization with $0.1 \%$ Triton $\mathrm{X}-100$ for $30 \mathrm{~min}$ at room temperature. After blocking with $20 \%$ normal goat serum in PBS 1x (Invitrogen, Germany), slices were incubated with primary antibodies overnight at $4^{\circ} \mathrm{C}$ followed by appropriate secondary antibody incubation for $1 \mathrm{~h}$ at room temperature. Both primary and secondary antibodies were diluted in $0.7 \%$ Carrageenan solution with $0.02 \% \mathrm{NaN}_{3}$ solution in PBS $1 \mathrm{x}$. The sections were counterstained with 4,6-diamidino-2-phenylindole (DAPI) (Invitrogen, Germany). Microglia activation was detected by using ionized calcium-binding adaptor molecular 1 (Iba1) (rabbit polyclonal, 1:200, Cat. $\mathrm{N}^{\circ}$ : 019-19741 (RRID: AB_839504), Wako, Germany), anti-Caspase-1 (p20) (mouse monoclonal, 1:500, Cat. $\mathrm{N}^{\circ}$ : AG-20B-0042-C100 (RRID: AB_2755041), AdipoGen), and anti-NLRP-3 inflammasome (rabbit polyclonal, $1: 200$, Cat. $\mathrm{N}^{\circ}$ : ab214185 (RRID: AB_2819003), Abcam, Germany) on sections of P8 rat pups. Because Iba-1 and NLRP-3 antibodies have the same host, we proceeded with sequential immunostaining. Immunohistochemistry were visualized by fluorescence microscopy AxioScan Z.1 (Zeiss, Germany), using a 20x objective. The images were analyzed using Zen 3.1 (Blue edition, Zeiss, Germany) and ImageJ. From each ipsilateral hemisphere, we analyzed the hippocampal areas (CA1, CA2, CA3, and CA4), ventroposterior thalamus nucleus, subthalamic nucleus, basolateral amygdaloid nucleus, and caudate putamen. We did not select the cortex area because of the amount of damage present. We selected the hippocampal regions (CA1, CA2, CA3, and CA4) as the most representative area due to the high expression of the markers used (Iba-1, Caspase-1, and NLRP-3). For Iba-1, we took a single image at $20 \mathrm{x}$ magnification to visualize the morphology of the microglia cells using a ZOE Fluorescent Cell Imager microscope (Bio-Rad, Germany). The contralateral side did not show differences in staining.

2.3. Magnetic Activated Cell Sorting (MACS) of CD11 b/cPositive Microglia. For microglia isolation, we proceeded to do magnetic cell sorting of CD11 b/c-positive microglia from the ligated brain hemispheres. Pups were sacrificed at different time points (Figure 1). To analyze the different alterations in phenotype polarization of microglia, we specifically isolated CD11 b/c-positive microglia ex vivo from rat brains at the previously mentioned time points. Due to the limitation of the technique regarding the amount of isolated cells, the severity of damage on the ipsilateral brains (especially at TP5-6), and because of the high mortality in our LPS/HI group, we proceeded to isolate and pool the brains for each condition and time point in order to get a workable amount of microglia cells with the highest yield required for 
an optimal RT-PCR of the full set of cytokines selected for the study. For vehicle and LPS, 5 full brains were pooled from each separate condition since they were not exposed to HI. For vehicle/HI and LPS/HI, a total of 10 ipsilateral hemispheres (most affected hemisphere after HI; [15, 17]) were pooled per condition and time point. The brains were mechanically dissociated in HBSS $1 x$ cold buffer. Myelinremoval beads were used following distributors instructions followed by magnetic separation on LS columns. For MACS, the obtained cell mixtures were washed with MACS buffer (PBS $1 x$ containing 0.5\% BSA and $0.2 \mathrm{mM}$ EDTA) and incubated with anti-CD11b/c coupled microbeads (Miltenyi Biotec, Germany) followed by magnetic separation on MS columns of the mini-MACS magnetic separation kit (Miltenyi Biotec, Germany) following distributor instructions. The total effluent (negative fraction), after removal of the column CD11 b/c-positive microglia, was eluted in a volume of $1 \mathrm{ml}$ MACS buffer. To investigate the purity of the magnetically separated cells, a small volume of the positive eluate was analyzed via flow cytometry (data not shown). The rest of separated positive CD11 b/c cells was used for real-time PCR as a unique sample for each treatment in each time point.

2.4. Real-Time PCR. RNA from isolated microglia was generated following the distributor instructions from RNeasy Mini Kit (QIAGEN, Germany). First strand complementary DNA was synthesized using $2 \mu \mathrm{g}$ of total RNA and TaqMan reverse transcription reagents (Applied Biosystems/Thermo Fisher Scientific, USA). The 96-well optical reaction plates were used to perform the PCR amplification. 40 cycles (each cycle at $94^{\circ} \mathrm{C}$ for $15 \mathrm{~s}$ and $60^{\circ} \mathrm{C}$ for $1 \mathrm{~min}$ ) were done using the StepOnePlus Real-Time PCR system (Applied Biosystems/Thermo Fisher Scientific, USA). Analysis was performed at different time points (TP1, TP2, TP3, TP4, TP5, and TP6) in our 4 predefined groups (vehicle, LPS, vehicle/ $\mathrm{HI}$, and LPS/HI). The PCR results of pro- and antiinflammatory cytokines were quantified by fluorogenic reporter oligonucleotide probes. Pro- and antiinflammatory markers as well as gene expression of the inflammasome cryopyrin (NLRP-3), including as well Caspase- 1 and $I L-18$ used in this study, are listed in Table 1 and were purchased from Thermo Fisher Scientific, Germany. Beta-actin was used as housekeeping gene. The results were normalized to the vehicle group. Generally, real-time PCR and detection were performed in duplicates (2-technical replicates). Target gene expression was quantified according to the $2^{\Delta \Delta \mathrm{CT}}$ method [46]. Fold change was used as "magnitude scale" to classify the extend of gene expression for the pro- and anti-inflammatory effects after the different treatments over the times analyzed. Figures were plotted as each condition per separate, including all time points together for the purpose of a better overview of the effect over time after the different treatments.

2.5. RNA Sequencing and Gene Set Analysis. RNA sequencing and gene set analysis were performed as previously described [43]. Ingenuity Pathway Analysis was performed on the significant genes comparing a condition of interest to other condi-
Table 1: Primer list used for the real-time PCR.

\begin{tabular}{lccc}
\hline Gene & Official symbol & Entrez gene ID & Product number \\
\hline Arg1 & Arg1 & 29221 & Rn00691090_m1 \\
$\beta$-Actin & Actb & 81822 & Rn00667869_m1 \\
CCL11 & Ccl11 & 29397 & Rn00569995_m1 \\
Caspase 1 & Casp1 & 25166 & Rn00562724_m1 \\
CD206 & Mrc1 & 291327 & Rn01487342_m1 \\
IL1beta & Il1b & 24494 & Rn00580432_m1 \\
IL4 & Il4 & 287287 & Rn99999010_m1 \\
IL6 & Il6 & 24498 & Rn01410330_m1 \\
IL18 & Il18 & 29197 & Rn01422083_m1 \\
IL12 & Il12a & 84405 & Rn00584538_m1 \\
iNOS & Nos2 & 24599 & Rn00561646_m1 \\
LIF & Lif & 60584 & Rn00573491_g1 \\
NLRP3 & Nlrp3 & 287362 & Rn04244621_m1 \\
TGF-beta & Tgfb1 & 59086 & Rn00572010_m1 \\
TNF-alpha & Tnf & 24835 & Rn01525859_g1 \\
\hline
\end{tabular}

tions (i.e., hypoxia alone samples vs LPS/HI alone samples). The data from upstream regulators were then plotted as a volcano plot ( $x$-axis activation, $z$-score, $y$-axis, and $-\log 10 p$ value). From 549 gene expression profiles for different regulators expressed in Veh/HI and LPS/HI at $24 \mathrm{~h}$ post HI, we preselected only upstream regulators, which are cytokines expressed in both condition. Highlighted in red are the most significant cytokines expressed in both groups, while those cytokines present only in the LPS/HI condition were highlighted in yellow. For our study, the following 11 cytokines were used: proinflammatory M1: IL1-beta, $I L-6, I L-12$, iNOS, and TNF-alpha and anti-inflammatory M2: Arg-1, CD206, CCL11, IL-4, LIF, and TGF-beta) [47-49].

2.6. Statistical Analysis. GraphPad Prism 6 (GraphPad Software, United States) was used to analyze and plot the data. For the RNA sequencing and gene set analysis, a $t$-test was performed to determine differential expression at the gene level $(p<0.05$, fold change \pm 2$)$.

\section{Results}

3.1. Transcriptomic Profiling of Presensitized Microglia after Hypoxia-Ischemia. Previous studies from our group demonstrated both a pro- and anti-inflammatory cytokine response $24 \mathrm{~h}$ post HI in our inflammation-sensitized HI model [43]. To further investigate the specific phenotype polarization of microglia in our study, we performed transcriptomic profiling of microglia cells in our model 24 hours post HI. At that time point, the cytokine profile showed a high regulation for genes with relevant function in microglia activation $[42,43]$. After RNAseq, we focused on a gene set of microglia cytokines, as this clustered gene set showed the most significant genes upregulated in the vehicle/HI and LPS/HI groups, as illustrated in a volcano plot (Figures 2(a) and 2(b)). From the full set of gene expression in both conditions (see Material and Methods), we highlighted in red the upstream regulators, which are cytokines presented in both groups, while 


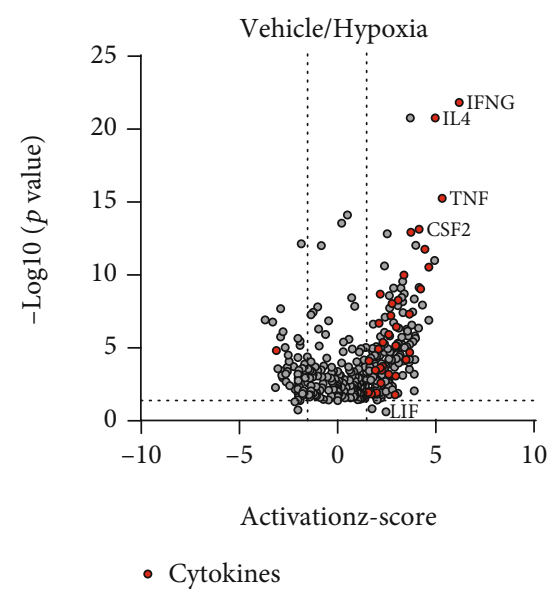

(a)

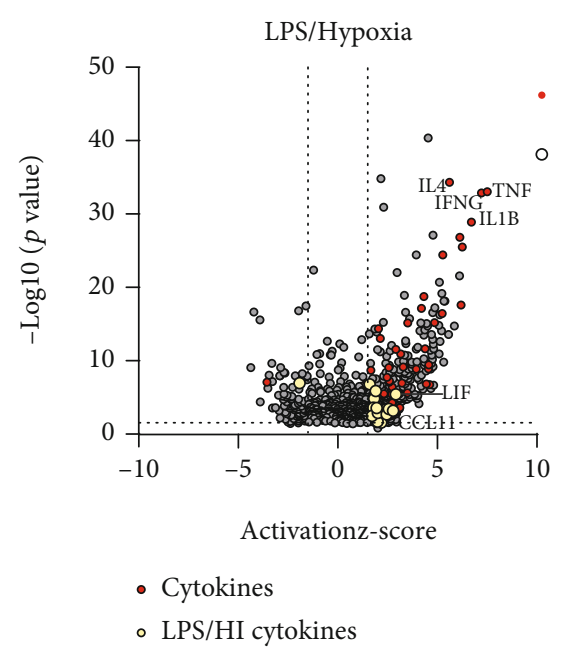

(b)

FIgURe 2: Significant upregulation of several cytokine genes following inflammation-sensitized HI injury. Positive CD11 b/c microglia were used for transcriptome analysis (RNA sequencing). Volcano plot ( $x$-axis activation, $z$-score, $y$-axis, and -log10 $p$ value) representing all upstream regulators in the LPS/HI and vehicle/HI groups (gray), where upstream cytokine regulators are highlighted in red for vehicle/ $\mathrm{HI}$ and LPS/HI group (a-b) and cytokines only present in the LPS/HI group are highlighted in yellow (b).

in yellow, only those expressed in the LPS/HI group are presented. In the present study, a total of 11 most significant genes from the cytokines cluster were selected in base of their function in microglia activation and inflammation $[25,31,50,51]$. For our study, the following cytokines were used: proinflammatory M1: IL1-beta, $I L-6, I L-12$, iNOS, and TNF-alpha and anti-inflammatory M2: Arg-1, CD206, CCL11, IL-4, LIF, and TGF-beta [47-49].

3.2. Time Dependency of Proinflammatory Genes in Microglia after LPS-Sensitized Hypoxic-Ischemic Brain Injury. To assess the inflammatory response in our LPS presensitized model before and after HI exposure, we compared gene expression profiles associated with proinflammatory cytokines in sorted CD11b/c microglia cells from pooled full brains from the vehicle and LPS alone groups, while the ipsilateral hemisphere brains were pooled and used for the vehicle/HI and LPS/HI groups [52]. We analyzed and quantified the gene expression levels of the following proinflammatory cytokines: IL-1beta, IL-6, IL-12, iNOS, and TNF-alpha. To determine the degree of inflammation in our model before exposure to HI, we analyzed microglia-associated cytokines before animals underwent carotid ligation and hypoxia. Our data showed a clear increase in the expression level for all the proinflammatory cytokines analyzed at TP1 (3.5 h before HI) (Figures 3(a)-3(e)), where iNOS showed an increase with a magnitude of 20.000-fold changes compared to the vehicle group, followed by a 100-fold change for IL1-beta, IL-12, and TNF-alpha compared to the vehicle group, while $I L-6$ showed still an 8 -fold change compared with the vehicle group.

At TP2, we observed a prevailing increase in the expression levels for all the proinflammatory cytokines analyzed. The LPS/HI group showed a clear upregulation compared to the vehicle group for all the proinflammatory cytokines analyzed (Figures 3(a)-3(e)). Interestingly, the LPS/HI group showed also a substantial increase in the expression level compared to the vehicle/HI group. We observed for iNOS a 10.000-fold change in its expression level compared to the vehicle/HI group (Figure 3(d)), followed by a 100fold change for IL-1beta compared to the same groups (Figure 3(a)). IL-6, IL-12, and TNF-alpha showed less increase in their gene expression (Figures 3(b), 3c, and $3(\mathrm{e}))$. Additionally, LPS treatment alone led to a substantial increase in the expression for all the cytokines tested compared to the expression level of the vehicle group (Figures 3(a)-3(e)): fold change: $i N O S>10.000$, IL - 1beta $>100$, TNF - alpha > 10, IL-6, and $I L-12>5$ ). The vehicle/HI group showed a slight increase in its expression, showing a 10 -fold change for $T N F-\alpha$ compared to the vehicle group (Figure $3(\mathrm{e})$ ). This observation was less pronouncing when we compared the vehicle/HI group to the LPS/HI group (Figure 3(e)).

At TP3, iNOS showed a 100-fold change in the LPS/ HI group compared to the vehicle group (Figure $3(\mathrm{e})$ ), while $I L-1$ beta and $I L-6$ showed a less pronounce change in their expression level (10-fold change) in the LPS/HI group compared to the vehicle group (Figures 3(b) and $3(\mathrm{~d}))$. The expression level of $I L-1$ beta and $I L-6$ in the LPS group showed a substantial decrease compared to the LPS/HI group (Figures 3(a), 3(b), and 3(d)). Interestingly, we observed an increase in the LPS/HI group compared to the vehicle/HI group, where IL-1beta showed a 1000 -fold change compared to the vehicle/HI. IL-6 and iNOS showed a slight increase of 10-fold change (Figures 3(a), 3(b), and 3(e)). IL-12 and TNF-alpha showed a decrease in the gene expression for all treatment groups compared at TP3 (Figures 3(c) and 3(e)). At TP4, TNF-alpha and $I L-12$ showed no changes in their expression level in the different groups analyzed (Figure 3(a), $3(\mathrm{c})$, and $3(\mathrm{e}))$. However, IL-1beta and iNOS showed a slight upregulation in all the groups compared to the 


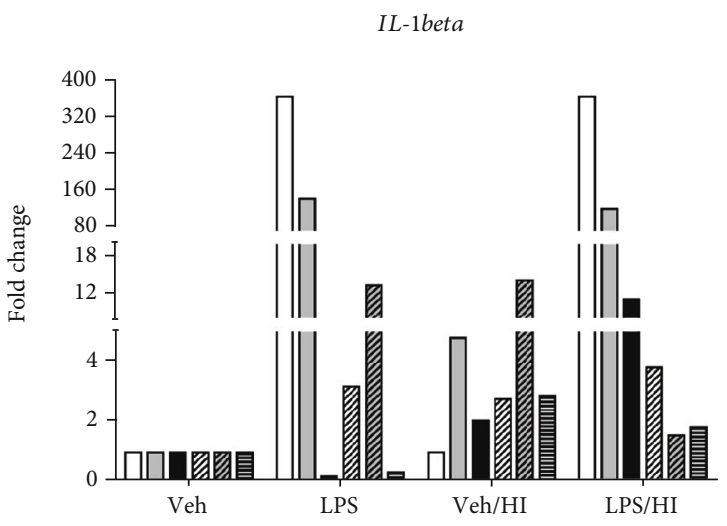

(a)

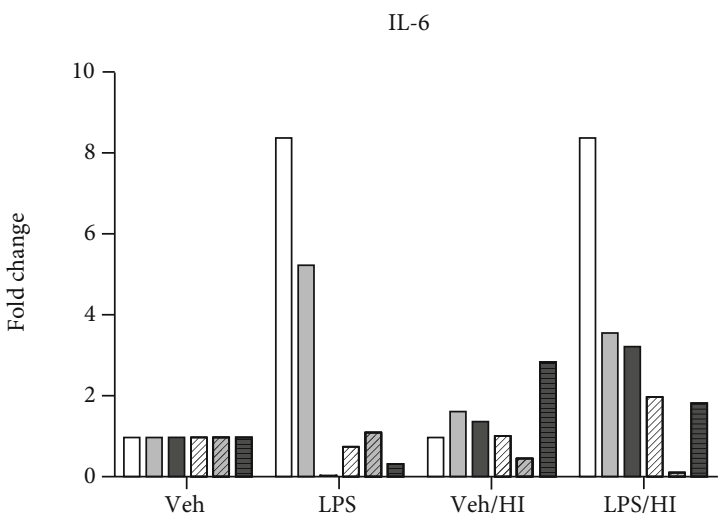

(b)

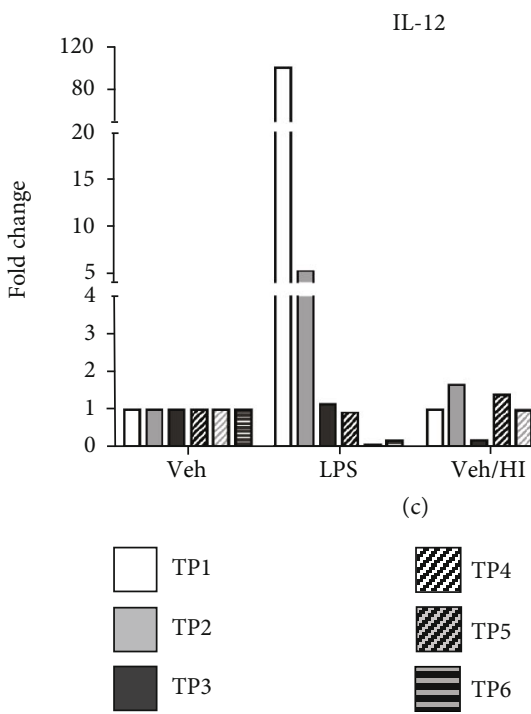

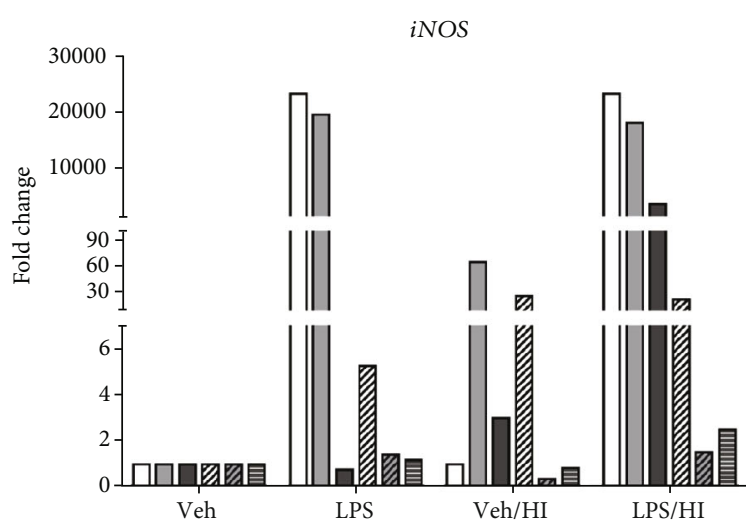

(d)

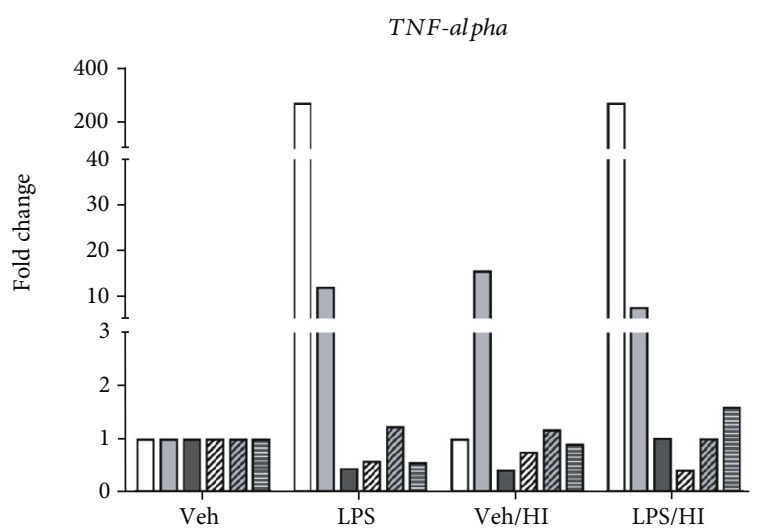

(e)

FIgURE 3: Proinflammatory cytokines expression of CD11 b/c microglia before and after inflammation-sensitized HI brain injury. A substantial upregulation immediately after inflammation-sensitized LPS compared to the vehicle group at TP1 was observed for all cytokines analyzed ( $3.5 \mathrm{~h}$ after i.p. LPS injection). (a) IL-1beta showed a remarkable upregulation at TP2, TP3, and TP5, with a slight decrease in the gene expression through TP4 and TP6. Only TP2, TP3, and TP5 showed a prevailing regulation of its expression in the group LPS/HI compared to the vehicle/HI. (b) IL-6 showed a similar upregulation pattern as IL-1beta with a slight increase after TP6, with a remarkable upregulation in the LPS/HI group compared to the vehicle/HI at TP2, TP3, and TP6. (c) IL-12 showed upregulation at TP2 and TP5 in the LPS/HI group compared to the vehicle/HI. (d) iNOS showed upregulation from TP2 to TP3 for vehicle/HI and LPS/HI groups. No changes in gene expression after TP5 were observed. (e) TNF-alpha showed a similar expression pattern as iNOS, with an increased at TP2, even for the LPS/HI compared to the vehicle/HI. No changes in the gene expression were found in the following TPs. Group sizes: vehicle $n=5$, LPS $n=5$, vehicle/HI $n=10$, and LPS/HI $n=10$ (microglia from the whole brains were pooled for vehicle or LPS, while 10 ipsilateral hemispheres were pooled for vehicle/HI or LPS/HI). 
vehicle group alone (Figure 3(a) and 3(d)). At TP5, ILlbeta showed a slight increase in the LPS and vehicle/ HI groups compared to the vehicle alone and LPS/HI group (Figure 3(a), fold change $>10$ ). IL-6 showed a downregulation in the expression level for LPS/HI compared to LPS (Figure 3(b)); and it was the only proinflammatory cytokine that at TP6 showed a slight increase on the vehicle/HI and the LPS/HI groups compared to the vehicle group (Figure 3(b)).

Our results at TP1 demonstrate that LPS sensitized microglia to a major inflammatory status and further following HI resulted in a constant inflammatory status that lasted over the time points studied, while the brains not presensitized showed a later regulation in their proinflammatory expression pattern.

3.3. Time Dependency of Anti-Inflammatory Genes in Microglia after LPS-Sensitized Hypoxic-Ischemic Brain Injury. The balance between pro- and anti-inflammatory mediators and their interactions is known to determine the magnitude of the inflammatory reaction. We assessed gene expression profiles associated with anti-inflammatory cytokines from sorted CD11b/c microglia from pooled full brains from the vehicle and LPS groups alone, while the ipsilateral hemisphere of brains were pooled and used from the vehicle/HI and LPS/HI groups [52]. As it was mentioned above, the degree of inflammation in our model before exposure to HI was analyzed from microglia-associated cytokines before animals underwent carotid ligation and hypoxia. Interestingly, the expression levels of classical anti-inflammatory cytokines (Figure 4(a)-4(f)) did not show as high expression levels as it was observed for the proinflammatory cytokines. At TP1, an increase for Arg-1, IL-4, and TGF-beta with a 40-fold change expression compared to the vehicle group alone was observed, while CCL11 and LIF showed a slight increase (fold change $>20$ and fold change $>10$, respectively) compared to the vehicle group. However, CD206 did not show any change in its expression level at this time point (Figure 4(b)).

As described before for TP1, a high gene expression for some anti-inflammatory cytokines immediately after LPS exposure demonstrates that microglia plays a dual role in the resolution of inflammation not only by the expression of proinflammatory cytokines but also by the regulation of anti-inflammatory cytokines $[36,53,54]$. At TP2, only L1F (Figure 4(e)) showed a 50-fold change increase in its expression level in the LPS/HI group compared to the vehicle group alone, while vehicle/HI group showed a 20-fold change increase compared to the vehicle group alone. CCL11 showed a slight increase in its expression for the LPS/HI and LPS groups compared to the vehicle group alone (Figure 4(c), fold change $>20$ ) and a 50 -fold change in its expression in the vehicle/HI group compared to the vehicle group alone. IL-4 showed a 50fold change increase in its expression levels only for the vehicle/HI group (Figure 4(b)). A slight upregulation was observed for TGF-beta with a 10-fold change for the same groups (Figure 4(f)). CD206 showed minimal changes in its gene expression at TP3 and TP6 in the LPS/HI group compared to the vehicle, LPS, and Veh/HI groups (Figure 4(b), fold change $<10$ ). On the other side, at TP4, Arg-1 showed a slight upregulation with a 50-fold change for the LPS/HI and vehicle/HI groups compared to the vehicle and LPS groups (Figure 4(a)). TGF-beta only showed a change at TP5 with a substantial downregulation in its gene expression for the LPS/HI group compared to the vehicle group (Figure 4(f)), while the LPS group compared to the vehicle group showed a moderately upregulation in its gene expression (Figure 4(f)).

3.4. Activation of the NLRP3 Inflammasome in Microglia after LPS-Sensitized Hypoxic-Ischemic Brain Injury. As NLRP-3 mediates Caspase-1 activation and the secretion of IL-1beta/IL-18; in both proinflammatory cytokines [55], leading to microglia activation, we analyzed NLRP-3, Caspase-1, IL-1beta, and IL-18 gene expressions in our model at early and later time points after HI. We did not find any activity of the inflammasome in microglia exposed to LPS before HI injury (Figure 5(a)). At TP3, NLPR-3 (Figure 5(a)) showed a slight upregulation in the vehicle/ HI and LPS/HI groups compared with the vehicle group (Figure 5(a)). We previously showed a significant upregulation in $N L R P-3$ gene expression $24 \mathrm{~h}$ post $\mathrm{HI}$ from the LPS/HI group compared to the vehicle group and a slight significant gene expression between vehicle/HI compared to the vehicle group [42]. In the present study, LPS, vehicle/HI, and LPS/HI groups showed a 5 -fold change increase compared to the vehicle group at TP5, while a 20 -fold change increase in gene expression between the LPS group compared to the LPS/HI group was observed. Interestingly, NLRP-3 expression maintained high in the LPS group at TP6 compared to the vehicle and LPS/HI groups (Figure 5(a)), while no changes were observed for the other treatment groups.

Caspase-1 showed a slight increase in its expression in the LPS groups compared with the vehicle at TP1. However, a 10-fold change was observed in the LPS/HI group compared with the vehicle at TP2. Interestingly, at TP4, LPS and vehicle/HI showed a 20 -fold change increase compared to the vehicle alone, while LPS/HI showed less increment of 10 -fold change. We also observed that vehicle/HI showed an expression level of 10-fold change compared to the LPS/HI group (Figure 5(b)).

Only at TP5, LPS/HI group showed a 20 -fold change compared to the other groups. TP6 did not show any change in gene expression for all the four groups analyzed. The timing observed for the gene expression for Caspase-1 followed a pattern associated with the pattern of the NLRP-3 inflammasome. The analysis for IL-1beta (previously described above) indeed showed similar patterns of activation as the NLRP-3 inflammasome (Figure 5(c)). The analysis of $I L-18$ showed similar time point expression as it was observed for NLRP-3 and Caspase-1. An increase of 80 -fold change was observed at TP1 for the LPS group compared to the vehicle group (Figure 5(d)), while LPS/HI showed an increase of 80 -fold change compared to the vehicle group as well as to the vehicle/HI group at TP2. At TP3, we did not observe major changes in its 


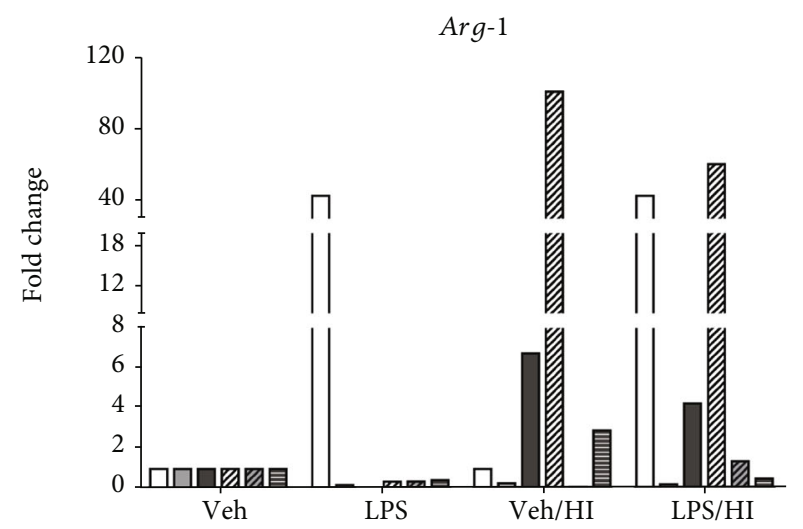

(a)

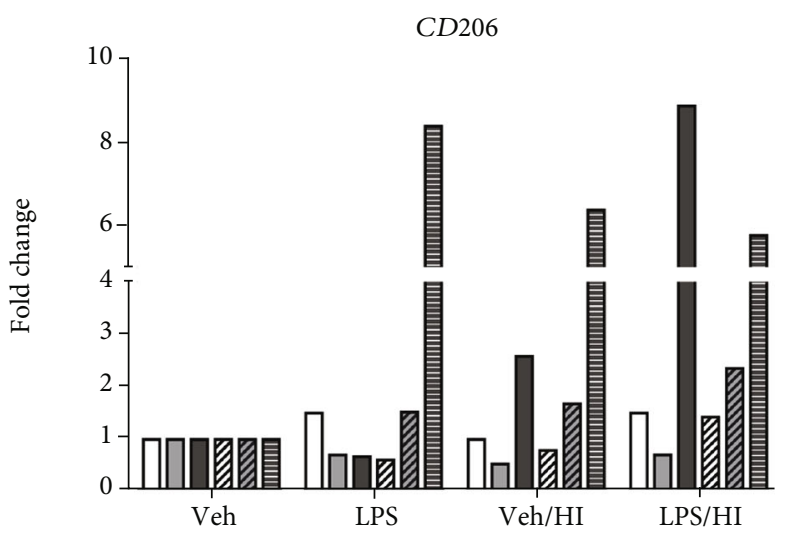

(b)

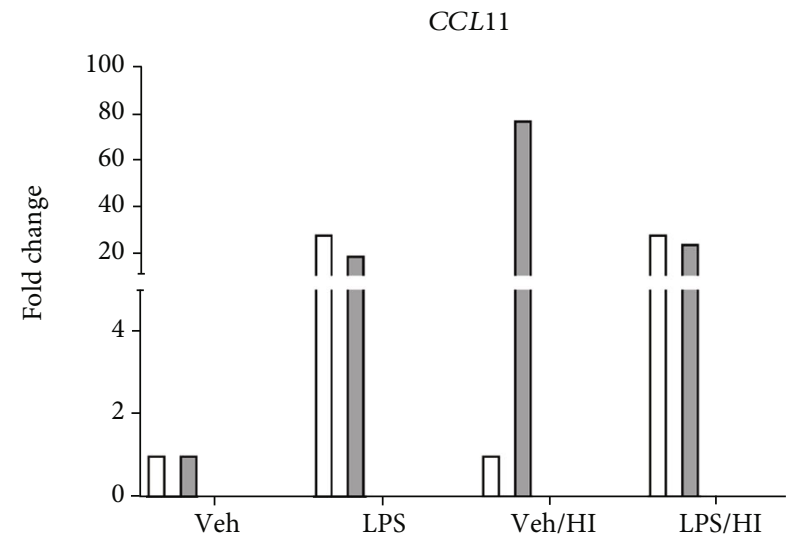

(c)

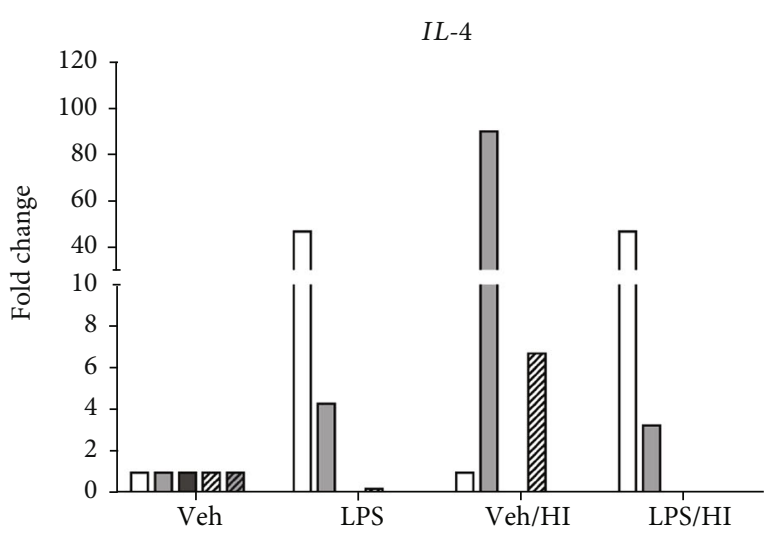

(d)

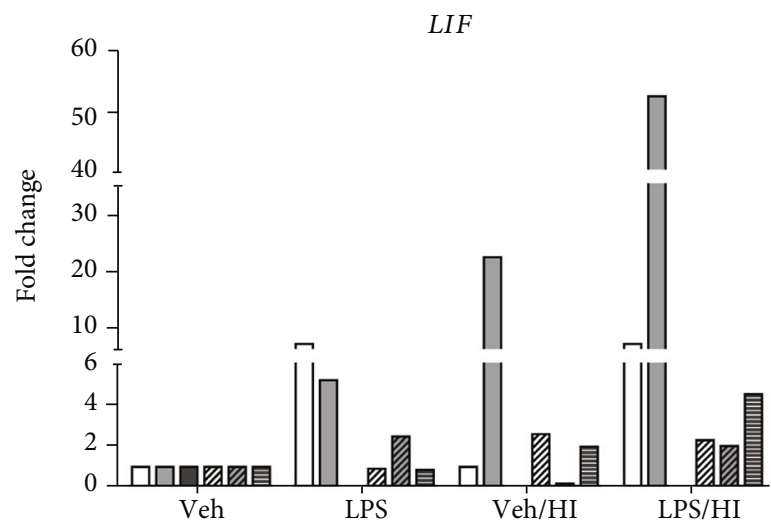

(e)

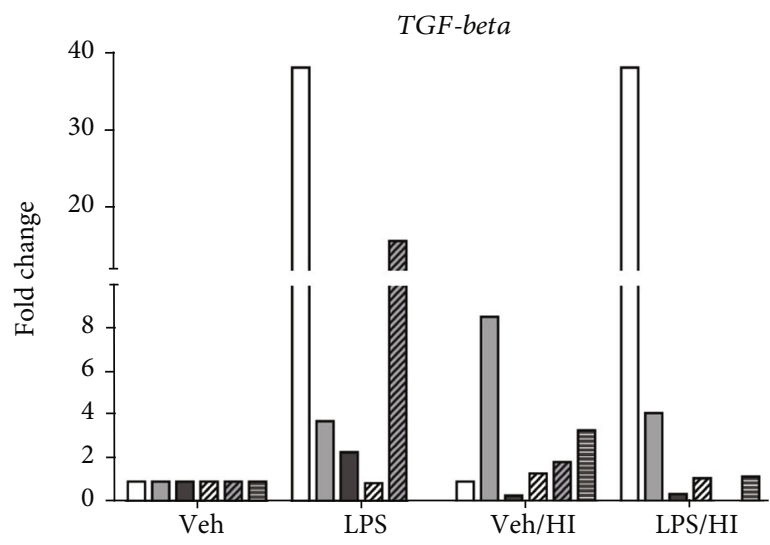

(f)

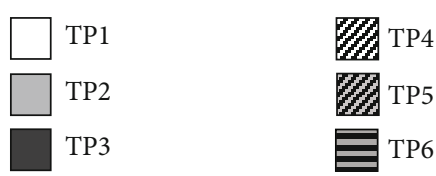

Figure 4: Anti-inflammatory cytokines expression of CD11 b/c microglia after inflammation-sensitized HI brain injury. A substantial upregulation immediately after inflammation-sensitized LPS compared to the vehicle group at TP1 was observed for Arg-1 (a), CCL11 (c), IL-4 (d), and TGF-beta (f) (3.5 h after i.p. LPS injection). (a) Arg-1 only showed upregulation at TP4 for the LPS/HI group compared to the vehicle/HI group. (b) CD206 showed upregulation at TP3 and at TP6, where only at TP3, LPS/HI showed a prevailing regulation against vehicle/HI group. (c-f) CCL11, IL-4, LIF, and TGF-beta only showed upregulation in gene expression immediately after HI at TP2. However, only CCL11, IL4, and LIF showed a change in the expression pattern between LPS/HI and vehicle/HI at TP2. Group sizes: vehicle $n=5$, LPS $n=5$, vehicle/HI $n=10$, and LPS/HI $n=10$ (microglia from the whole brains were pooled for vehicle or LPS, while 10 ipsilateral hemispheres were pooled for vehicle/HI or LPS/HI). 


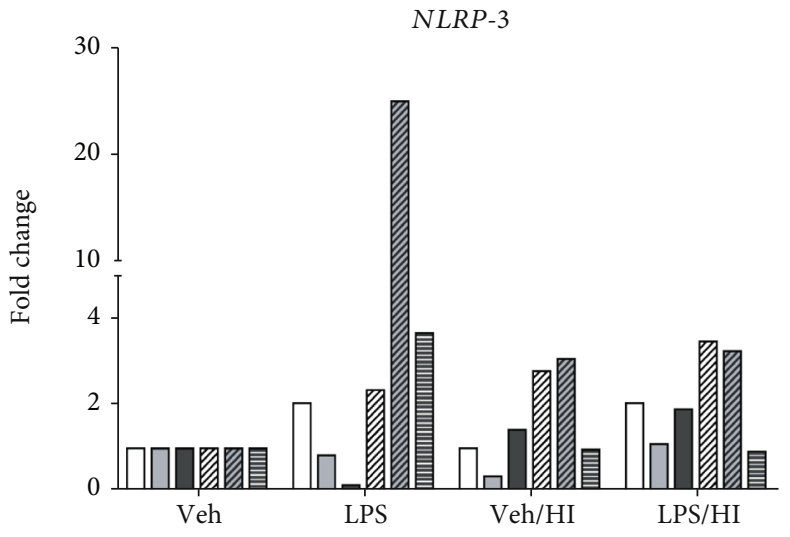

(a)

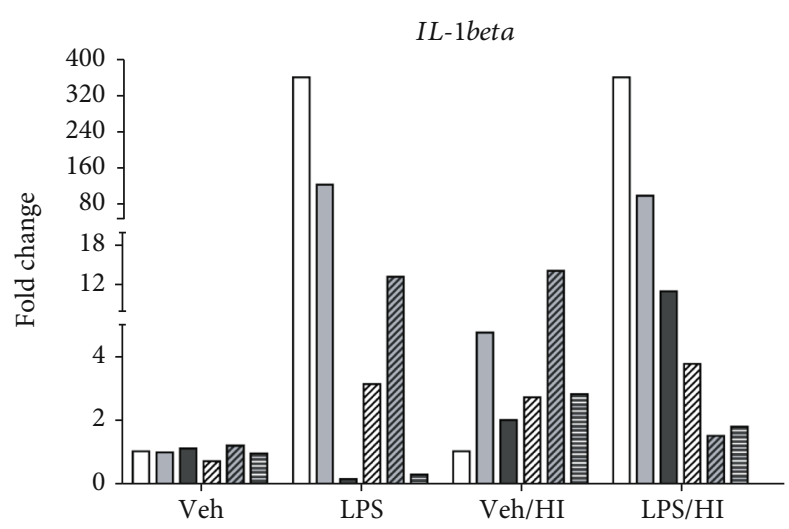

(c)

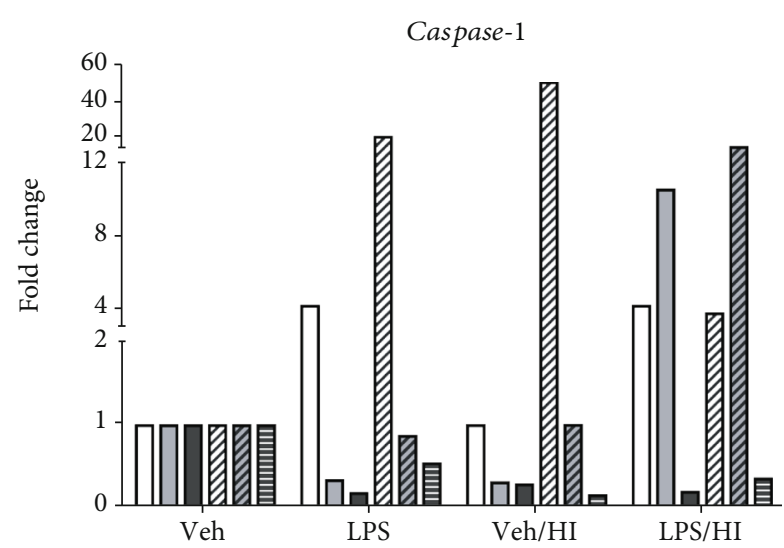

(b)

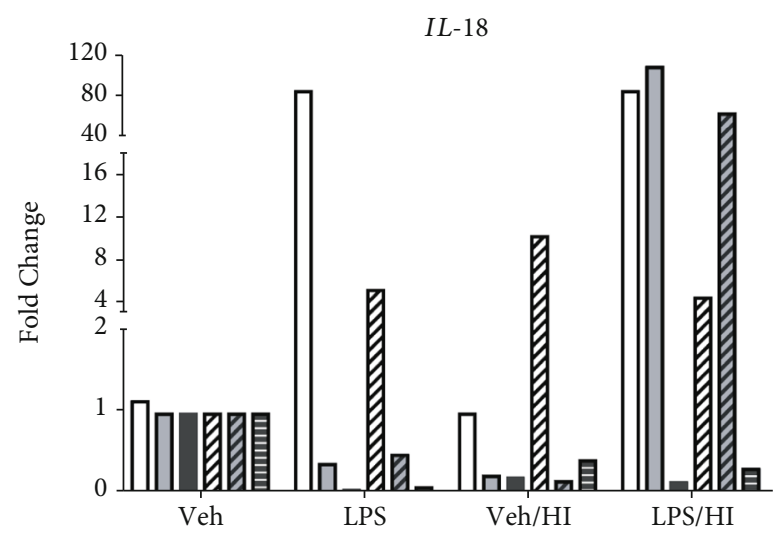

(d)

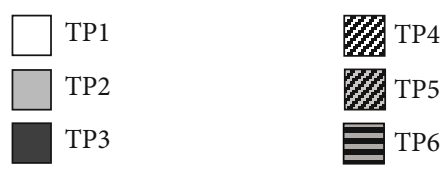

Figure 5: NLRP-3, Caspase-1, IL-1beta, and $I L-18$ gene expressions of CD11 b/c microglia after inflammation-sensitized HI brain injury. A substantial upregulation immediately after inflammation-sensitized LPS compared to the vehicle group at TP1 was observed for Caspase-1 (b), IL-1beta (c), and IL-18 (d) (3.5 h after i.p. LPS injection). (a) NLRP-3 gene expression showed upregulation from TP3 to TP6. At TP5, a substantial increase in the vehicle/HI group compared to the LPS/HI group was observed. (b) Caspase-1 showed a similar activation pattern as NLRP-3 with upregulation already at TP2 for the LPS/HI group compared to the other groups. (c) $I L-1 b e t a$ and (d) $I L-18$ showed an upregulation over the time analyzed and prevailing activation comparable to NLRP-3 gene expression. Group sizes: vehicle $n=5$, LPS $n$ $=5$, vehicle/HI $n=10$, and LPS/HI $n=10$ (microglia from the whole brains were pooled for vehicle or LPS, while 10 ipsilateral hemispheres were pooled for vehicle/HI or LPS/HI).

expression. However, at TP4, LPS/HI, vehicle/HI, and LPS showed an increase compared to the vehicle group alone (Figure 5(d)). Only LPS/HI showed an 80-fold change increase compared to the vehicle/HI, LPS, and vehicle groups at TP5. We did not observe major changes at TP6 for any of the groups analyzed.

\subsection{Hippocampal NLRP3 Inflammasome and Microglia} Activation after LPS-Sensitized Hypoxic-Ischemic Brain Injury. We previously demonstrated that the upregulated NLRP3 expression $24 \mathrm{~h}$ after HI (TP4 in our study) was particularly significant in the hippocampus and cortex of the rats from the LPS/HI group compared to the vehicle group [42]. Using immunohistochemistry staining for NLRP3 and the microglia marker Iba- 1 in brain slices from TP4
(Figure 6(a), yellow square (area analyzed in b, c, and d), and Figures $6(\mathrm{~b})-6(\mathrm{~d})$ ), we showed a strong positive staining in the hippocampus area for the LPS/HI, vehicle/HI, and LPS compared to the vehicle group. We observed severe cortical and hippocampal injury in the ipsilateral side of the brain in the LPS/HI group, which corroborated our observation from the previous work on 7-day postinsult brains with a predominant lesion for the same group [15, 17]. We observed a strong staining of Iba- 1 and NLRP 3 in the 4 fields from the hippocampus area (CA1, CA2, CA3, and CA4), ventroposterior thalamus nucleus, subthalamic nucleus, basolateral amygdaloid nucleus, and caudate putamen in the LPS/HI group (Figure 6(a) (yellow asterisk) and Figure 6(b)) with a clear staining for activated microglia (Figure 6(c)). The vehicle/HI group showed the most 
(a) Iba-1/Dapi
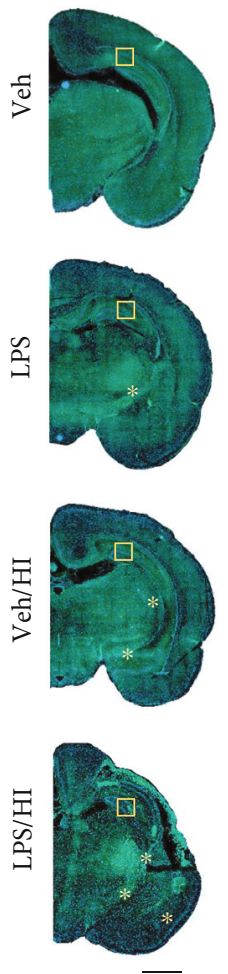

(e)
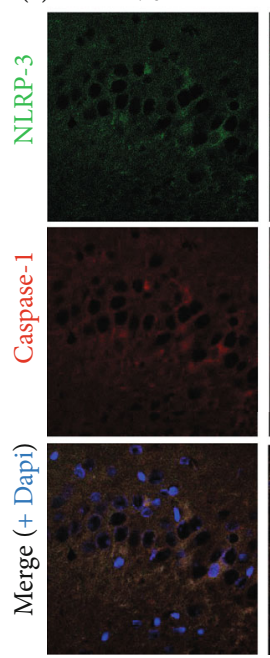

(b) Iba-1
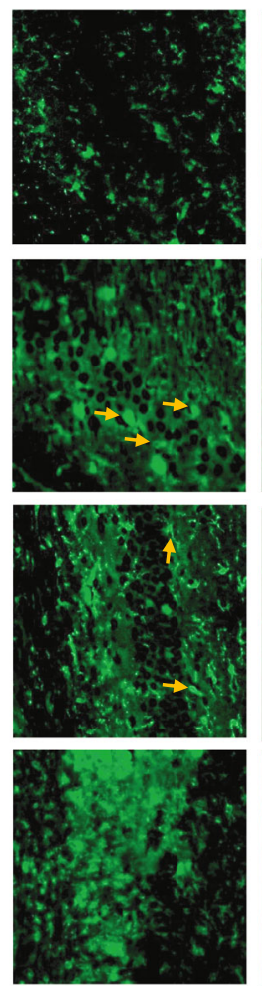

LPS
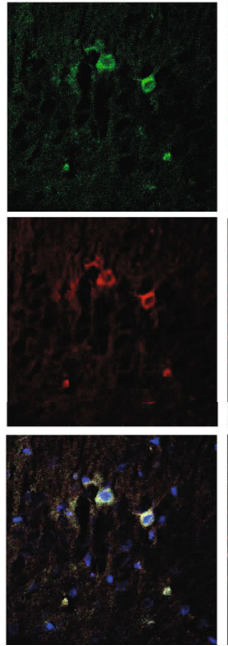

(c) Iba-1 Zoom
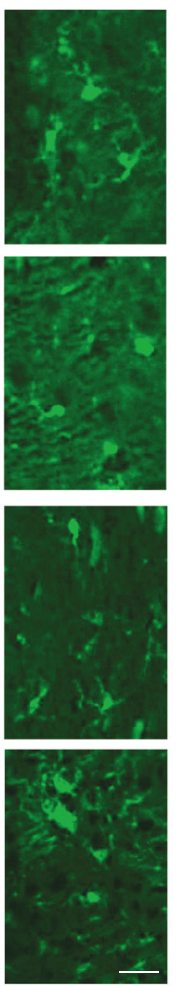

Veh/HI
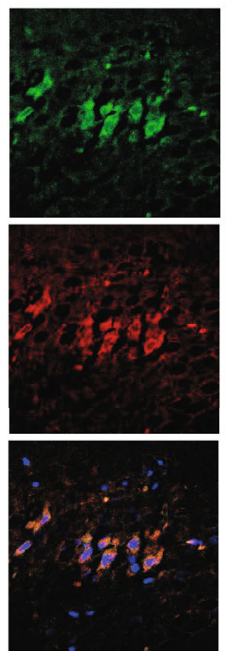

(d) NLRP-3
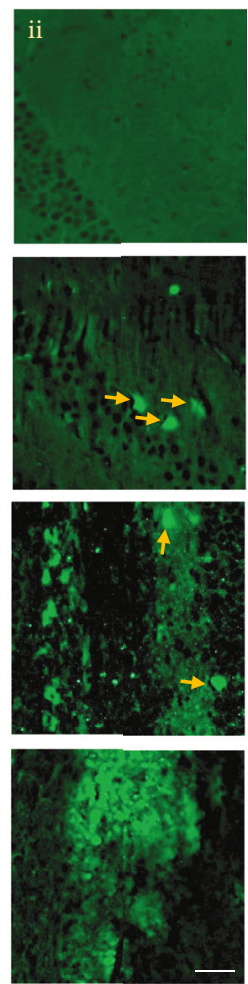

LPS/HI

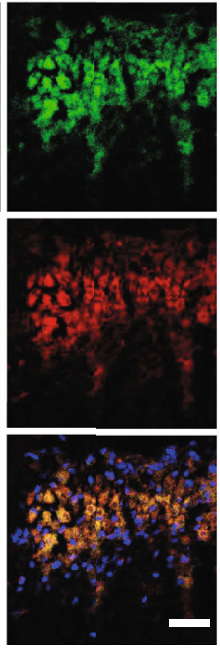

FIgURE 6: Immunohistochemistry of brain slices (bregma -3.8) at TP4. (a-c) Microglia marker Iba-1 in green and nucleus marker Dapi in blue. (d) NLRP-3 in green. (a) We observed at TP4 a severe lesion in the LPS/HI group compared to the vehicle or LPS groups alone. Yellow squares represent the hippocampal area analyzed in (b) and (d). Yellow asterisks represent other areas with immunopositive staining for Iba1 and NLRP-3. We observed for the LPS/HI group, NLRP-3-positive staining in the ventroposterior thalamus nucleus, subthalamic nucleus, basolateral amygdaloid nucleus, and caudate putamen, while for the vehicle/HI group, positive staining was only found in the ventroposterior thalamus nucleus and subthalamic nucleus. LPS group alone only showed positive NLRP-3 staining in the ventroposterior thalamus nucleus. (b) Iba-1 staining of the hippocampal area showed an increase in microglia cells with an increase in microgliosis in the LPS/HI group. (c) Magnification of the area in (b), showing the degree of polarization in microglia cells, with a clear, ramified morphology (inactivated microglia) in the vehicle group, to an amoeboid morphology (activated microglia) in the LPS/HI group. (d) NLRP-3-immunopositive staining of the hippocampal area with a remarkable increase of NLRP-3-positive cells in the same area where a strong microgliosis was observed in the LPS/HI group. Yellow arrows point cells positive for Iba-1 and NLRP-3. (e) Double-immunostaining shows colocalization in the hippocampus area for NLRP-3 (green) and Caspase-1 (red) and merge (+nuclear marker, Dapi in blue), with an increase in the amount of positive staining in the LPS/HI group. Scale bar: (a) $500 \mu \mathrm{m}$, (b, d-e) $100 \mu \mathrm{m}$, and (c) $20 \mu \mathrm{m}$. 
reduced area with Iba-1-positive staining (Figure 6(b), yellow arrows), with a variety of microglia polarization (Figure 6(c)) and much less positive staining for NLRP-3 in the CA4 hippocampus, ventroposterior thalamus nucleus, and subthalamic nucleus (Figure 6(c), yellow arrows). The LPS group showed few Iba-1-positive cells in a less activated state (Figure 6(b)-6(c), yellow arrows) and few positive NLRP-3 cells in the hippocampus, especially areas CA2 and CA4, and in the ventroposterior thalamus nucleus (Figure 6(d), yellow arrows). The vehicle group showed few Iba-1-positive microglia cells with a more ramified morphology and no positive staining for NLRP-3 (Figures 6(b)-6(d)). Due to the severity of the lesion after HI, we could not analyze the cortex area at TP 5. Most of the positive NLRP-3 staining were in the same or proximal to niches of positive microglia cells staining (Figure 3(d), yellow square and yellow arrows). However, we observed NLRP-3-positive staining in other cells, likely hippocampal neurons or motor neurons (data not shown). We also observed in immunohistochemistry from brain sections from TP4 a strong colocalization between NLRP-3 inflammasome and Caspase-1 in the hippocampus area (Figure 6(e)). LPS/HI and vehicle/HI showed a strong staining for NLRP-3 and Caspase- 1 in the 4 field from the hippocampus, while LPS group showed few immunopositive cells for NLRP-3 and Caspase-1 (Figure 6(e)). We did not observe immunostaining in the vehicle group.

\section{Discussion}

The risk of developing HIE is significantly increased with the combination of preinfection/inflammation and a potential birth asphyxiating condition, compared to either alone [8, 56-60]. The mechanism by which preinflammation increases brain vulnerability to HI is complex.

A number of observational studies suggest an increased risk of neurodevelopmental impairment following infection/inflammation in newborns [8, 10, 23, 49]. Here, we demonstrate a biological basis possibly explaining the severe adverse outcome in inflammation-sensitized asphyxiated neonates. It is commonly believed that similar to the initiation of inflammation, resolution of inflammation is an active process in which inflammation-resolving cells like microglia and their cytokines are pivotal for the termination of the inflammatory response [61]; however, as much as inflammation is a pivotal process in fighting off many threatening conditions, when it is unresolved, it forms the basis of a wide range of persistent/chronic diseases and secondary damage mediated by the inflammatory response constantly disrupting the return to homeostasis [61].

In the present study, we used bacterial LPS; a component of the cell walls of gram-negative bacteria. Although grampositive organisms are the most common cause of antenatal/postnatal infection in high resources settings, gramnegative infection/inflammation is increasing in frequency in the low- and middle-resource settings, surpassing grampositive organisms as the leading causative pathogen in neonatal infection/inflammation associated HIE [62, 63]. In models of LPS sensitization, the mechanism mainly starts with the activation of toll-like receptor 4 (TLR4) which trig- gers many self-perpetuating pathways, among them are microglia activation/proliferation and release of proinflammatory cytokines $[31,64]$. The Vannucci model is the most established animal model for newborn HI brain injury in rodents that led to translational clinical trials and the establishment of $\mathrm{TH}$ to reduce mortality and morbidities following perinatal asphyxia [65]. However, clinically up to $50 \%$ of all cooled newborns from the large randomized controlled trials did not benefit from cooling therapy [4]. It has been demonstrated that the beneficial effect of $\mathrm{TH}$ depends on the time window during which the treatment starts after an insult. Immediately after injury or with a delay of not more than $6 \mathrm{~h}, \mathrm{TH}$ is neuroprotective after moderate $\mathrm{HI}$; however, the neuroprotective effect is unfavorable when the treatment is delayed or even after a severe insult $[16,66,67]$. Currently, it has been shown that $\mathrm{TH}$ is not beneficial in the setting of low- and middle-income countries and strengthens our findings of lack of TH neuroprotection in our LPS-sensitized HI rodent model $[14,15,17]$. However, the molecular mechanism by which LPS presensitization occurs and why HT is not beneficial in particular cases are not known yet.

Previous publications from our laboratory demonstrated a high expression of proinflammatory cytokines and the NLRP-3 inflammasome only at the $24 \mathrm{~h}$ time point after the insult in our LPS presensitized model of HI brain injury $[42,43]$. However, the time-dependent temporal expression of pro- and anti-inflammatory cytokines in this model is of main interest, as it might give us the possibility to understand why $\mathrm{TH}$ is not neuroprotective in this model and at what time points potential alternative treatments might be beneficial.

We propose that microglia play a key role in the severity of brain injury following LPS-sensitized HI [15, 43], with a predominant proinflammatory (M1) and antiinflammatory (M2) phenotype in the first hours after LPS, exacerbating the microglia response after a mild HI injury compared to HI alone. However, the effect observed in a not presensitized brain showed a delay in the inflammatory resolution by microglia cells. We highlight the involvement of the NLRP3 inflammasome in the inflammatory process of our brain injury model preexposed with LPS and following $\mathrm{HI}$, with a remarkable lesion at early time points that could explain the severity of the LPS-sensitized HI brain injury observed at 7 -day post insult $[15,17]$.

We observed a predominant upregulation of proinflammatory genes, like $I L-1$ beta, $I L-6$, iNOS, and TNF-alpha and anti-inflammatory genes, like Arg1, CCL11, IL-1, and TGFbeta immediately after LPS sensitization. We demonstrate strong microglia activation and proinflammatory gene expression that exacerbates its reaction after a second insult (like HI). Anti-inflammatory genes showed minor reactions over the times analyzed. Interestingly, HI alone showed a delay in the proinflammatory expression pattern compared to LPS/HI, while anti-inflammatory cytokine expression remained unchanged. Microglia are immune cells which permanently reside in the CNS [25]. Following CNS pathologies, microglia activation happens as a first immune response for the removal of threatening compounds [28, 29, 61, 68, 69]. However, microglia responses often fail in 
the removal of threats or even result in an escalating effect of a vicious cycle of unresolved local cytotoxic inflammation, which might override the beneficial effect of these cells [36, 51, 53, 61, 70].

Proinflammatory cytokines like IL-1beta, IL-6, IL-12, iNOS, and TNF-alpha are likely one of the first immune mediators which show an upregulation just after an injury. They strongly enhance inflammatory responses and have profound effects on blood brain barrier (BBB) permeability, cell death via programed necrosis, glial activation, immune cell recruitment, neuron excitotoxicity damage, and ultimately in neurodegeneration [51, 71-81]. It has been demonstrated that neuronal self-injury after exposure to LPS could be alleviated by using specific cytokine blockers, as it was already shown for IL-1beta [78]. On the other side, some cytokines like IL-6 has a dual function, where high levels early after HI insults are associated with adverse HIE outcomes; a secondary peak later after HIE was associated with better outcomes by regulating expression levels of IL-1beta and TNF-alpha $[56,82-85]$ and by its ability to promote neuronal differentiation of neural stem progenitor cells (NSPCs) and promote angiogenesis [86].

The most intensively studied inflammasome is NLRP-3, and it has been shown to be involved in many neurological diseases in adults [87]. NLRP-3 inflammasome mediates Caspase-1 activation in response to cellular damage, with consequently activation and secretion of proinflammatory cytokines like IL-1beta and IL-18 [88, 89]. Stimuli like LPS/HI trigger inflammasome assembly and activation of pyroptosis, a form of cell death $[78,90,91]$. It has previously been demonstrated that after inhibition of NLRP-3, using MCC950 inhibitor, there is a reduction of pyroptosis in injured rat brain with HIE [92]. After infection/inflammation and $\mathrm{HI}$, regulatory pathway involvement might explain how LPS preexposure significantly increases the vulnerability of the newborn brain to a mild hypoxic-ischemic event $[89,92,93]$.

While our study shows a pronounced proinflammatory expression and the activation of NLRP-3 inflammasome, anti-inflammatory cytokines did not show a major upregulation over the time. Only after LPS sensitization, a high increase in the anti-inflammatory gene expression was observed, except for CD206 and LIF. However, after HI, CCL11, IL-4, LIF, and TGF-beta showed a continued upregulation that did not last over time for all the treatments. Anti-inflammatory cytokines regulate the differentiation of specific T-cells (TH17) [94], maturation and normal homeostasis of microglia cells [95], detoxification by removing excessive nitrogen [96, 97], promotion of neurogenesis, and axonal repair mechanisms [98]. Interestingly, our RNA sequence data point out other cytokines regulated after LPS/ $\mathrm{HI}$ that gained increased interest over the past years due to their anti-inflammatory function. It has been demonstrated in a neonatal mouse model of cerebral ischemia, CCL11 levels are also upregulated after cerebral ischemia, which results in promoting migration of NSPCs in these mice [99, 100]. Another interesting cytokine was Leukemia Inhibitory Factor (LIF), where it has been shown in a neonatal mouse HI model, a reduction of astrogliosis and microgliosis after intranasal LIF administration, showing preservation of myelin [48].
We clearly observed in our study that a single LPS dose triggers a cascade of inflammatory responses, presensitizing the brain to a major inflammatory state, making it vulnerable to a second insult compared to $\mathrm{HI}$ alone. TH has become the standard treatment for neonates with HIE. However, the preexistence of an inflammatory status before $\mathrm{HI}$ could reduce the therapeutic window for optimal neuroprotection after TH and could answer why neonates with presensitized brain injury do not benefit from the treatment. We show with our results a time window for the activation of microglia and secretion of different cytokines before and after HI and how a presensitized brain increases the activation of microglia cells into a predominantly M1 phenotype.

The transition between M1/M2 microglia could potentially lead to novel treatment options and/or improve the actual TH. Depletion of anti-inflammatory cytokines has shown impaired oligodendrocytes maturation and subsequent hypomyelination of gray matter tracts as well as postnatal loss of cortical interneurons [101], while enhancing their secretion promotes the phagocytic activity and migration of microglia cells through apoptotic cells, explaining the relevant function of early expression in the clearing of dead cells after an insult $[102,103]$.

Our study presents some limitations. We could not differentiate between gender and individual subjects because of the technique chosen to isolate microglia. However, we randomized each group between genders, and each group had an equal amount of animals with the same gender. However, further studies are needed to understand the differences between genders in this model. Another limitation is that we have analyzed gene expression patterns and not protein levels. This remarks the importance of further experiments at the protein level in order to confirm our results.

Understanding the role of cytokines in the evolution of neonatal brain injury, as well as the dynamic nature of cytokine release after a hypoxic-ischemic insult, is a promising avenue for identifying biomarkers of ongoing brain injury in newborns with antenatal/postnatal infection/inflammation following $\mathrm{HI}$, especially those that do not show an improved outcome after TH [84, 104-107].

The actual challenge in neonatal asphyxia is to find the line between the beneficial aspects of neuroinflammation following the insult to allow neuroprotection and regeneration while at the same time minimizing its harmful effects in the newborn CNS. Identified key factors that could be used for an early identification of infants with an extensive and potentially damaging neuroinflammatory response of those with moderate inflammation would present new options for a more individualized therapeutic approach in neonatal asphyxia as well as determine the impact of $\mathrm{TH}$ and/or to find other neuroprotective treatments, with a potential improvement in future clinical studies that will help to further improve outcome in asphyxiated newborns, especially in countries with high perinatal infection and perinatal asphyxia rates.

\section{Conclusion}

Our results demonstrate that microglia are early key mediators of the inflammatory response and inflammation 
sensitization exacerbates the inflammatory response following $\mathrm{HI}$, polarizing into a predominant proinflammatory M1 phenotype in the early hours post HI. This may explain why antenatal/postnatal infection-/inflammation-correlated HIE shows an unfavorable outcome compared to HIE alone and that cooling is not beneficial in the context of inflammation-sensitized HIE. Additionally, we demonstrate the involvement of the NLRP3 inflammasome, highlighting one potential regulatory pathway in our model. These findings will help us to better understand the complex inflammatory mechanisms and could be the start point to study microglia polarization to a more beneficial M2 phenotype at specific time points during the insult. However, more research in the topic is needed in the molecular mechanism that gives us in the future the possibility to early intervene and offer new treatment options that will help to further improve outcome in asphyxiated newborns, especially in developing countries with higher infection rates.

\section{Data Availability}

The raw data supporting the conclusion of this article will be made available by the authors, without undue reservation, to any qualified researcher.

\section{Ethical Approval}

All animal experiments were performed in accordance with the Animal Protection Committee of the North RhineWestphalia State Environment Agency (LANUV), Germany.

\section{Conflicts of Interest}

The author(s) declare(s) that they have no conflicts of interest.

\section{Authors' Contributions}

MEB and HS planned and designed the study. MEB, HS, $\mathrm{MM}$, and EM performed the animal experiments. MEB, YS, and HS performed real-time PCR analysis. MEB, MM, and EM performed tissue analysis. MEB, DP, and HS analyzed the data. MEB, UFM, and HS wrote and corrected the manuscript.

\section{Acknowledgments}

We would like to thank the Light Microscopy Facility (LMF) and the people from the animal house from the Deutsche Zentrum für Neurodegenerative Erkrankungen (DZNE) Bonn, Germany. This study was funded by the Deutsche Forschungsgemeinschaft (DFG, German Research Foundation, Project number 422493683) and the Federal Ministry of Education and Research (BMBF, grant number 13GW0297).

\section{References}

[1] S. K. Dhillon, C. A. Lear, R. Galinsky et al., "The fetus at the tipping point: modifying the outcome of fetal asphyxia,"
The Journal of Physiology, vol. 596, no. 23, pp. 5571-5592, 2018.

[2] D. R. Ophelders, R. Gussenhoven, L. Klein et al., "Preterm brain injury, antenatal triggers, and therapeutics: timing is key," Cell, vol. 9, no. 8, p. 1871, 2020.

[3] P. Greco, G. Nencini, I. Piva et al., "Pathophysiology of hypoxic-ischemic encephalopathy: a review of the past and a view on the future," Acta Neurologica Belgica, vol. 120, no. 2, pp. 277-288, 2020.

[4] S. E. Jacobs, M. Berg, R. Hunt et al., "Cooling for newborns with hypoxic ischaemic encephalopathy," Cochrane Database of Systematic Reviews, vol. 1, p. CD003311, 2013.

[5] S. Aslam, T. Strickland, and E. J. Molloy, "Neonatal encephalopathy: need for recognition of multiple etiologies for optimal management," Frontiers in Pediatrics, vol. 7, p. 142, 2019.

[6] H. Wang, C. A. Liddell, M. M. Coates et al., "Global, regional, and national levels of neonatal, infant, and under- 5 mortality during 1990-2013: a systematic analysis for the Global Burden of Disease Study 2013," The Lancet, vol. 384, no. 9947, pp. 957-979, 2014.

[7] E. Kasdorf and J. M. Perlman, "Hyperthermia, inflammation, and perinatal brain injury," Pediatric Neurology, vol. 49, no. 1, pp. 8-14, 2013.

[8] K. B. Nelson and J. K. Grether, "Potentially asphyxiating conditions and spastic cerebral palsy in infants of normal birth weight," American Journal of Obstetrics and Gynecology, vol. 179, no. 2, pp. 507-513, 1998.

[9] M. Martinez-Biarge, J. Diez-Sebastian, C. J. Wusthoff, E. Mercuri, and F. M. Cowan, "Antepartum and intrapartum factors preceding neonatal hypoxic-ischemic encephalopathy," Pediatrics, vol. 132, no. 4, pp. e952-e959, 2013.

[10] Y. W. Wu, G. J. Escobar, J. K. Grether, L. A. Croen, J. D. Greene, and T. B. Newman, "Chorioamnionitis and cerebral palsy in term and near term infants," Journal of the American Medical Association, vol. 290, 2003.

[11] J. Nair and V. H. S. Kumar, "Current and Emerging Therapies in the Management of Hypoxic Ischemic Encephalopathy in Neonates," Children, vol. 5, no. 7, p. 99, 2018.

[12] J. Danladi and H. Sabir, "Perinatal infection: a major contributor to efficacy of cooling in newborns following birth asphyxia," International Journal of Molecular Sciences, vol. 22, no. 2, p. 707, 2021.

[13] M. Hakobyan, K. P. Dijkman, S. Laroche et al., "Outcome of infants with therapeutic hypothermia after perinatal asphyxia and early-onset sepsis," Neonatology, vol. 115, no. 2, pp. 127133, 2019.

[14] S. Thayyil, S. Pant, P. Montaldo et al., "Hypothermia for moderate or severe neonatal encephalopathy in low-income and middle-income countries (HELIX): a randomised controlled trial in India, Sri Lanka, and Bangladesh," The Lancet Global Health, vol. 9, no. 9, pp. e1273-e1285, 2021.

[15] D. Osredkar, M. Thoresen, E. Maes, T. Flatebo, M. Elstad, and H. Sabir, "Hypothermia is not neuroprotective after infection-sensitized neonatal hypoxic-ischemic brain injury," Resuscitation, vol. 85, no. 4, pp. 567-572, 2014.

[16] H. Sabir, E. Scull-Brown, X. Liu, and M. Thoresen, "Immediate hypothermia is not neuroprotective after severe hypoxia-ischemia and is deleterious when delayed by 12 hours in neonatal rats," Stroke, vol. 43, no. 12, pp. 33643370, 2012. 
[17] D. Osredkar, H. Sabir, M. Falck et al., "Hypothermia does not reverse cellular responses caused by lipopolysaccharide in neonatal hypoxic-Ischaemic brain injury," Developmental Neuroscience, vol. 37, no. 4-5, pp. 390-397, 2015.

[18] H. J. Bonestroo, C. J. Heijnen, F. Groenendaal, F. van Bel, and C. H. Nijboer, "Development of cerebral gray and white matter injury and cerebral inflammation over time after inflammatory perinatal asphyxia," Developmental Neuroscience, vol. 37, no. 1, pp. 78-94, 2015.

[19] T. Wood, D. Osredkar, M. Puchades et al., "Treatment temperature and insult severity influence the neuroprotective effects of therapeutic hypothermia," Scientific Reports, vol. 6, no. 1, p. 23430, 2016.

[20] M. Falck, D. Osredkar, E. Maes et al., "Hypothermic neuronal rescue from infection-sensitised hypoxic-Ischaemic brain injury is pathogen dependent," Developmental Neuroscience, vol. 39, no. 1-4, pp. 238-247, 2017.

[21] M. Falck, D. Osredkar, T. R. Wood et al., "Neonatal systemic inflammation induces inflammatory reactions and brain apoptosis in a pathogen-specific manner," Neonatology, vol. 113, no. 3, pp. 212-220, 2018.

[22] S. Eklind, C. Mallard, A. L. Leverin et al., "Bacterial endotoxin sensitizes the immature brain to hypoxic-ischaemic injury," European Journal of Neuroscience, vol. 13, no. 6, pp. 11011106, 2001.

[23] S. Eklind, C. Mallard, P. Arvidsson, and H. Hagberg, "Lipopolysaccharide induces both a primary and a secondary phase of sensitization in the developing rat brain," Pediatric Research, vol. 58, no. 1, pp. 112-116, 2005.

[24] S. Eklind, H. Hagberg, X. Wang et al., "Effect of lipopolysaccharide on global gene expression in the immature rat brain," Pediatric Research, vol. 60, no. 2, pp. 161-168, 2006.

[25] B. Fleiss, C. J. Tann, V. Degos et al., "Inflammation-induced sensitization of the brain in term infants," Developmental Medicine and Child Neurology, vol. 57, Suppl 3, pp. 17-28, 2015.

[26] S. Nair, K. S. Sobotka, P. Joshi et al., "Lipopolysaccharideinduced alteration of mitochondrial morphology induces a metabolic shift in microglia modulating the inflammatory response in vitro and in vivo," Glia, vol. 67, no. 6, pp. 10471061, 2019.

[27] C. Mallard, M. E. Tremblay, and Z. S. Vexler, "Microglia and neonatal brain injury," Neuroscience, vol. 405, pp. 68-76, 2019.

[28] Q. Li and B. A. Barres, "Microglia and macrophages in brain homeostasis and disease," Nature Reviews. Immunology, vol. 18, no. 4, pp. 225-242, 2018.

[29] K. Saijo and C. K. Glass, "Microglial cell origin and phenotypes in health and disease," Nature Reviews. Immunology, vol. 11, no. 11, pp. 775-787, 2011.

[30] Q. Q. Yang and J. W. Zhou, "Neuroinflammation in the central nervous system: symphony of glial cells," Glia, vol. 67, no. 6, pp. 1017-1035, 2019.

[31] D. H. Baltazar, R. Nadella, A. B. Bonilla et al., "Does lipopolysaccharide-based neuroinflammation induce microglia polarization?," Folia Neuropathologica, vol. 58, no. 2, pp. 113-122, 2020.

[32] V. Stratoulias, J. L. Venero, M. E. Tremblay, and B. Joseph, "Microglial subtypes: diversity within the microglial community," The EMBO Journal, vol. 38, no. 17, article e101997, 2019.
[33] C. T. Jiang, W. F. Wu, Y. H. Deng, and J. W. Ge, "Modulators of microglia activation and polarization in ischemic stroke (review)," Molecular Medicine Reports, vol. 21, no. 5, pp. 2006-2018, 2020.

[34] M. Lalancette-Hebert, G. Gowing, A. Simard, Y. C. Weng, and J. Kriz, "Selective ablation of proliferating microglial cells exacerbates ischemic injury in the brain," The Journal of Neuroscience, vol. 27, no. 10, pp. 2596-2605, 2007.

[35] S. Tsuji, E. Di Martino, T. Mukai et al., "Aggravated brain injury after neonatal hypoxic ischemia in microgliadepleted mice," Journal of Neuroinflammation, vol. 17, no. 1, p. 111, 2020.

[36] V. Chhor, T. Le Charpentier, S. Lebon et al., "Characterization of phenotype markers and neuronotoxic potential of polarised primary microglia _in vitro_," Brain, Behavior, and Immunity, vol. 32, pp. 70-85, 2013.

[37] L. Lian, Y. Zhang, L. Liu et al., "Neuroinflammation in ischemic stroke: focus on microRNA-mediated polarization of microglia," Frontiers in Molecular Neuroscience, vol. 13, article 612439, 2021.

[38] J. J. Volpe, "Brain injury in premature infants: a complex amalgam of destructive and developmental disturbances," The Lancet, vol. 8, no. 1, pp. 110-124, 2009.

[39] S. A. Back, A. Riddle, and M. M. McClure, "Maturationdependent vulnerability of perinatal white matter in premature birth," Stroke, vol. 38, no. 2, pp. 724-730, 2007.

[40] M. B. Ystgaard, Y. Sejersted, E. M. Loberg, E. Lien, A. Yndestad, and O. D. Saugstad, "Early upregulation of NLRP3 in the brain of neonatal mice exposed to hypoxiaischemia: no early neuroprotective effects of NLRP3 deficiency," Neonatology, vol. 108, no. 3, pp. 211-219, 2015.

[41] A. Gustin, M. Kirchmeyer, E. Koncina et al., "NLRP3 inflammasome is expressed and functional in mouse brain microglia but not in astrocytes," PLoS One, vol. 10, no. 6, article e0130624, 2015.

[42] M. Serdar, K. Kempe, M. Rizazad et al., "Early proinflammatory microglia activation after inflammationsensitized hypoxic-ischemic brain injury in neonatal rats," Frontiers in Cellular Neuroscience, vol. 13, p. 237, 2019.

[43] M. Serdar, K. Kempe, R. Herrmann et al., "Involvement of CXCL1/CXCR2 during microglia activation following inflammation-sensitized hypoxic-ischemic brain injury in neonatal rats," Frontiers in Neurology, vol. 11, article 540878, 2020.

[44] B. S. Reinboth, C. Köster, H. Abberger et al., "Endogenous hypothermic response to hypoxia reduces brain injury: implications for modeling hypoxic-ischemic encephalopathy and therapeutic hypothermia in neonatal mice," Experimental Neurology, vol. 283, pp. 264-275, 2016.

[45] M. Serdar, J. Herz, K. Kempe et al., "Fingolimod protects against neonatal white matter damage and long-term cognitive deficits caused by hyperoxia," Brain, Behavior, and Immunity, vol. 52, pp. 106-119, 2016.

[46] K. J. Livak and T. D. Schmittgen, "Analysis of relative gene expression data using real-time quantitative PCR and the $2^{-\Delta \Delta}{ }_{-} C_{-}$Tethod," Methods (San Diego, Calif)., vol. 25, no. 4, pp. 402-408, 2001.

[47] B. Parajuli, H. Horiuchi, T. Mizuno, H. Takeuchi, and A. Suzumura, "CCL11 enhances excitotoxic neuronal death by producing reactive oxygen species in microglia," Glia, vol. 63 , no. 12 , pp. 2274-2284, 2015. 
[48] J. Lin, Y. Niimi, M. G. Clausi, H. D. Kanal, and S. W. Levison, "Neuroregenerative and protective functions of leukemia inhibitory factor in perinatal hypoxic-ischemic brain injury," Experimental Neurology, vol. 330, article 113324, 2020.

[49] H. Hagberg, C. Mallard, D. M. Ferriero et al., "The role of inflammation in perinatal brain injury," Nature Reviews. Neurology, vol. 11, no. 4, pp. 192-208, 2015.

[50] A. Ferro, Y. S. S. Auguste, and L. Cheadle, "Microglia, cytokines, and neural activity: unexpected interactions in brain development and function," Frontiers in Immunology, vol. 12, article 703527, 2021.

[51] J. M. Ziebell and M. C. Morganti-Kossmann, "Involvement of pro- and anti-inflammatory cytokines and chemokines in the pathophysiology of traumatic brain injury," Neurotherapeutics: The Journal of the American Society for Experimental NeuroTherapeutics, vol. 7, no. 1, pp. 22-30, 2010.

[52] S. Kannan, H. Dai, R. S. Navath et al., "Dendrimer-based postnatal therapy for neuroinflammation and cerebral palsy in a rabbit model," Science translational medicine, vol. 4, no. 130, article 130ra46, 2012.

[53] N. Hellström Erkenstam, P. L. Smith, B. Fleiss et al., "Temporal characterization of microglia/macrophage phenotypes in a mouse model of neonatal hypoxic-ischemic brain injury," Frontiers in Cellular Neuroscience, vol. 10, p. 286, 2016.

[54] R. Franco and D. Fernandez-Suarez, "Alternatively activated microglia and macrophages in the central nervous system," Progress in Neurobiology, vol. 131, pp. 65-86, 2015.

[55] M. S. J. Mangan, E. J. Olhava, W. R. Roush, H. M. Seidel, G. D. Glick, and E. Latz, "Targeting the NLRP3 inflammasome in inflammatory diseases," Nature Reviews. Drug Discovery, vol. 17, no. 8, pp. 588-606, 2018.

[56] D. D. Jenkins, L. G. Rollins, J. K. Perkel et al., "Serum cytokines in a clinical trial of hypothermia for neonatal hypoxic-ischemic encephalopathy," Journal of Cerebral Blood Flow and Metabolism, vol. 32, no. 10, pp. 1888-1896, 2012.

[57] A. I. Bartha, A. Foster-Barber, S. P. Miller et al., "Neonatal encephalopathy: association of cytokines with MR spectroscopy and outcome," Pediatric Research, vol. 56, no. 6, pp. 960-966, 2004.

[58] L. F. Chalak, P. J. Sanchez, B. Adams-Huet, A. R. Laptook, R. J. Heyne, and C. R. Rosenfeld, "Biomarkers for severity of neonatal hypoxic-ischemic encephalopathy and outcomes in newborns receiving hypothermia therapy," The Journal of Pediatrics, vol. 164, no. 3, pp. 468-74 e1, 2014.

[59] C. J. Tann, K. A. Martinello, S. Sadoo et al., "Neonatal encephalopathy with group B streptococcal disease worldwide: systematic review, investigator group datasets, and meta-analysis," Clinical Infectious Diseases, vol. 65, suppl_2, pp. S173-S189, 2017.

[60] C. I. K. J. Rousset, P. Olivier, S. Chalon, P. Gressens, and E. Saliba, "Antenatal bacterial endotoxin sensitizes the immature rat brain to postnatal excitotoxic injury," Journal of Neuropathology and Experimental Neurology, vol. 67, no. 10, pp. 994-1000, 2008.

[61] B. Fleiss, J. Van Steenwinckel, C. Bokobza, K. I. Shearer, E. Ross-Munro, and P. Gressens, "Microglia-mediated neurodegeneration in perinatal brain injuries," Biomolecules, vol. 11, no. 1, p. 99, 2021.

[62] A. K. Zaidi, D. Thaver, S. A. Ali, and T. A. Khan, "Pathogens associated with sepsis in newborns and young infants in developing countries," The Pediatric Infectious Disease Journal, vol. 28, no. 1, pp. S10-S18, 2009.

[63] A. Zea-Vera and T. J. Ochoa, "Challenges in the diagnosis and management of neonatal sepsis," Journal of Tropical Pediatrics, vol. 61, no. 1, pp. 1-13, 2015.

[64] K. A. Martinello, C. Meehan, A. Avdic-Belltheus et al., "Acute LPS sensitization and continuous infusion exacerbates hypoxic brain injury in a piglet model of neonatal encephalopathy," Scientific Reports, vol. 9, no. 1, p. 10184, 2019.

[65] J. E. Rice 3rd, R. C. Vannucci, and J. B. Brierley, "The influence of immaturity on hypoxic-ischemic brain damage in the rat," Annals of Neurology, vol. 9, no. 2, pp. 131-141, 1981.

[66] M. Thoresen, J. Tooley, X. Liu et al., "Time is brain: starting therapeutic hypothermia within three hours after birth improves motor outcome in asphyxiated newborns," Neonatology, vol. 104, no. 3, pp. 228-233, 2013.

[67] O. Iwata, S. Iwata, J. S. Thornton et al., "'Therapeutic time window" duration decreases with increasing severity of cerebral hypoxia-ischaemia under normothermia and delayed hypothermia in newborn piglets," Brain Research, vol. 1154, pp. 173-180, 2007.

[68] M. Prinz and J. Priller, "Microglia and brain macrophages in the molecular age: from origin to neuropsychiatric disease," Nature Reviews. Neuroscience, vol. 15, no. 5, pp. 300-312, 2014.

[69] E. R. Matarredona, R. Talaveron, and A. M. Pastor, "Interactions between neural progenitor cells and microglia in the subventricular zone: physiological implications in the neurogenic niche and after implantation in the injured brain," Frontiers in Cellular Neuroscience, vol. 12, p. 268, 2018.

[70] S. Fumagalli, C. Perego, F. Pischiutta, E. R. Zanier, and M. G. De Simoni, "The ischemic environment drives microglia and macrophage function," Frontiers in Neurology, vol. 6, p. 81, 2015.

[71] F. Liu and L. D. Mccullough, "Inflammatory responses in hypoxic ischemic encephalopathy," Acta Pharmacologica Sinica, vol. 34, no. 9, pp. 1121-1130, 2013.

[72] A. D. Greenhalgh, S. David, and F. C. Bennett, "Immune cell regulation of glia during CNS injury and disease," Nature Reviews Neuroscience, vol. 21, no. 3, pp. 139-152, 2020.

[73] H. W. Yao and C. Y. Kuan, "Early neutrophil infiltration is critical for inflammation-sensitized hypoxic-ischemic brain injury in newborns," Journal of Cerebral Blood Flow \& Metabolism, vol. 40, no. 11, pp. 2188-2200, 2020.

[74] P. L. Smith, A. Mottahedin, P. Svedin et al., "Peripheral myeloid cells contribute to brain injury in male neonatal mice," Journal of Neuroinflammation, vol. 15, no. 1, 2018.

[75] X. S. Yang, T. L. Yi, S. Zhang et al., "Hypoxia-inducible factor-1 alpha is involved in RIP-induced necroptosis caused by in vitro and in vivo ischemic brain injury," Scientific Reports, vol. 7, no. 1, p. 5818, 2017.

[76] S. W. Kim, H. Lee, H. K. Lee, I. D. Kim, and J. K. Lee, "Neutrophil extracellular trap induced by HMGB1 exacerbates damages in the ischemic brain," Acta Neuropathologica Communications, vol. 7, no. 1, p. 94, 2019.

[77] M. G. Frank, M. D. Weber, L. K. Fonken, S. A. Hershman, L. R. Watkins, and S. F. Maier, "The redox state of the alarmin HMGB1 is a pivotal factor in neuroinflammatory and 
microglial priming: a role for the NLRP3 inflammasome," Brain, Behavior, and Immunity, vol. 55, pp. 215-224, 2016.

[78] A. Savard, M. E. Brochu, M. Chevin, C. Guiraut, D. Grbic, and G. Sebire, "Neuronal self-injury mediated by IL-1 $\beta$ and MMP-9 in a cerebral palsy model of severe neonatal encephalopathy induced by immune activation plus hypoxia-ischemia," Journal of Neuroinflammation, vol. 12, no. 1, p. 111, 2015.

[79] B. Viviani, M. Boraso, N. Marchetti, and M. Marinovich, "Perspectives on neuroinflammation and excitotoxicity: a neurotoxic conspiracy?," Neurotoxicology, vol. 43, pp. 1020, 2014.

[80] J. Scheller, A. Chalaris, D. Schmidt-Arras, and S. Rose-John, "The pro- and anti-inflammatory properties of the cytokine interleukin-6," Biochimica et Biophysica Acta, vol. 1813, no. 5, pp. 878-888, 2011.

[81] A. O'Garra and N. Arai, “The molecular basis of T helper 1 and T helper 2 cell differentiation," Trend in Cell Biology, vol. 10, no. 12, pp. 542-550, 2000.

[82] A. Róka, G. Bekő, J. Halász et al., "Changes in serum cytokine and cortisol levels in normothermic and hypothermic term neonates after perinatal asphyxia," Inflammation Research, vol. 62, no. 1, pp. 81-87, 2013.

[83] K. Leifsdottir, H. Mehmet, S. Eksborg, and E. Herlenius, "Fasligand and interleukin- 6 in the cerebrospinal fluid are early predictors of hypoxic-ischemic encephalopathy and longterm outcomes after birth asphyxia in term infants," Journal of Neuroinflammation, vol. 15, no. 1, p. 223, 2018.

[84] A. Bajnok, L. Berta, C. Orbán et al., "Distinct cytokine patterns may regulate the severity of neonatal asphyxia-an observational study," Journal of Neuroinflammation, vol. 14, no. 1, p. 244, 2017.

[85] H. Aly, M. T. Khashaba, M. El-Ayouty, O. El-Sayed, and B. M. Hasanein, "IL-1 $\beta$, IL- 6 and TNF- $\alpha$ and outcomes of neonatal hypoxic ischemic encephalopathy," Brain \& Development, vol. 28, no. 3, pp. 178-182, 2006.

[86] C. Meng, J. C. Zhang, R. L. Shi, S. H. Zhang, and S. Y. Yuan, "Inhibition of interleukin- 6 abolishes the promoting effects of pair housing on post-stroke neurogenesis," Neuroscience, vol. 307, pp. 160-170, 2015.

[87] T. Strowig, J. Henao-Mejia, E. Elinav, and R. Flavell, "Inflammasomes in health and disease," Nature, vol. 481, no. 7381, pp. 278-286, 2012.

[88] Y. Yang, H. Wang, M. Kouadir, H. Song, and F. Shi, "Recent advances in the mechanisms of NLRP3 inflammasome activation and its inhibitors," Cell Death \& Disease, vol. 10, no. 2, p. 128, 2019.

[89] N. Kelley, D. Jeltema, Y. Duan, and Y. He, “The NLRP3 inflammasome: an overview of mechanisms of activation and regulation," International Journal of Molecular Sciences, vol. 20, no. 13, p. 3328, 2019.

[90] S. Kesavardhana, R. K. S. Malireddi, and T. D. Kanneganti, "Caspases in cell death, inflammation, and Pyroptosis," Annual Review of Immunology, vol. 38, no. 1, pp. 567-595, 2020.

[91] P. Yu, X. Zhang, N. Liu, L. Tang, C. Peng, and X. Chen, "Pyroptosis: mechanisms and diseases," Signal Transduction and Targeted Therapy, vol. 6, no. 1, p. 128, 2021.

[92] Y. Lv, B. Sun, X. X. Lu et al., "The role of microglia mediated pyroptosis in neonatal hypoxic-ischemic brain damage," Bio- chemical and Biophysical Research Communications, vol. 521, no. 4, pp. 933-938, 2020.

[93] C. A. Dinarello, "Immunological and inflammatory functions of the Interleukin-1 family," Annual Review of Immunology, vol. 27, no. 1, pp. 519-550, 2009.

[94] H. Qin, L. Wang, T. Feng et al., "TGF-beta promotes Th17 cell development through inhibition of SOCS3," Journal of immunology, vol. 183, no. 1, pp. 97-105, 2009.

[95] A. Von Ehr, A. Attaai, N. Neidert et al., "Inhibition of microglial TGF $\beta$ signaling increases expression of Mrc1," Frontiers in Cellular Neuroscience, vol. 14, p. 66, 2020.

[96] C. Thornton, A. A. Baburamani, A. Kichev, and H. Hagberg, "Oxidative stress and endoplasmic reticulum (ER) stress in the development of neonatal hypoxic-ischaemic brain injury," Biochemical Society Transactions, vol. 45, no. 5, pp. 1067-1076, 2017.

[97] M. Douglas-Escobar and M. D. Weiss, "Hypoxic-ischemic encephalopathy," JAMA Pediatrics, vol. 169, no. 4, pp. 397403, 2015.

[98] J. Krystofova, P. Pathipati, J. Russ, A. Sheldon, and D. Ferriero, "The arginase pathway in neonatal brain hypoxia-ischemia," Developmental Neuroscience, vol. 40, no. 56, pp. 437-450, 2019.

[99] F. Wang, N. Baba, Y. Shen et al., "CCL11 promotes migration and proliferation of mouse neural progenitor cells," Stem Cell Research \& Therapy, vol. 8, no. 1, p. 26, 2017.

[100] S. Lieschke, B. Zechmeister, M. Haupt et al., "CCL11 differentially affects post-stroke brain injury and neuroregeneration in mice depending on age," Cell, vol. 9, no. 1, 2020.

[101] B. Spittau, N. Dokalis, and M. Prinz, "The role of TGF $\beta$ signaling in microglia maturation and activation," Trends in Immunology, vol. 41, no. 9, pp. 836-848, 2020.

[102] S. Yi, X. Jiang, X. Tang et al., "IL-4 and IL-10 promotes phagocytic activity of microglia by up-regulation of TREM2," Cytotechnology, vol. 72, no. 4, pp. 589-602, 2020.

[103] X. Zhao, H. Wang, G. Sun, J. Zhang, N. J. Edwards, and J. Aronowski, "Neuronal interleukin-4 as a modulator of microglial pathways and ischemic brain damage," The Journal of Neuroscience, vol. 35, no. 32, pp. 11281-11291, 2015.

[104] S. Perrone, M. D. Weiss, F. Proietti et al., "Identification of a panel of cytokines in neonates with hypoxic ischemic encephalopathy treated with hypothermia," Cytokine, vol. 111, pp. 119-124, 2018.

[105] M. I. O’Dea, L. A. Kelly, E. McKenna et al., “Altered cytokine endotoxin responses in neonatal encephalopathy predict MRI outcomes," Frontiers in Pediatrics, vol. 9, 2021.

[106] L. A. Kelly, M. I. O'Dea, Z. Zareen et al., “Altered inflammasome activation in neonatal encephalopathy persists in childhood," Clinical and Experimental Immunology, vol. 205, no. 1, pp. 89-97, 2021.

[107] I. Lingam, A. Avdic-Belltheus, C. Meehan et al., "Serial blood cytokine and chemokine mRNA and microRNA over $48 \mathrm{~h}$ are insult specific in a piglet model of inflammationsensitized hypoxia-ischaemia," Pediatric Research, vol. 89, no. 3, pp. 464-475, 2021. 